\title{
Oxygen-Centered Tetradecaimido Hexatantalum Cluster Complexes
}

\author{
Jamin L. Krinsky, Laura L. Anderson, ${ }^{\dagger}$ John Arnold,* and Robert G. Bergman* \\ Department of Chemistry, University of California, Berkeley, California, 94720 \\ ${ }^{\dagger}$ Department of Chemistry, University of California, Irvine, California, 92697
}

\section{Supporting Information}

\begin{abstract}
Table of Contents

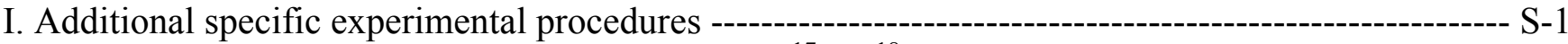

II. Electrospray Mass Spectra for 3 and 4, Including ${ }^{17} \mathrm{O}-,{ }^{18} \mathrm{O}-$ Enriched Samples ----------------------- S-4

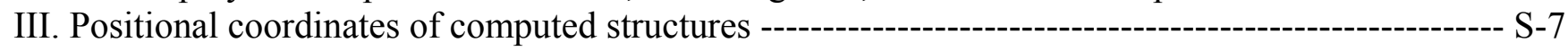

\section{Additional specific experimental procedures.}

$(\mathbf{P h N})_{14}$ Ta $_{6} \mathbf{O}$ (2), from compound 1. Compound 1 (100 mg, $0.190 \mathrm{mmol}, 1$ equiv) was dissolved in $1 \mathrm{~mL}$ of benzene. Aniline ( $89 \mathrm{mg}, 0.95 \mathrm{mmol}, 5$ equiv) was treated with $0.6 \mu \mathrm{L}(0.03 \mathrm{mmol}, 0.16$ equiv) of degassed water via microsyringe and the resulting mixture was dissolved in $0.5 \mathrm{~mL}$ benzene. The two solutions were mixed, and the reaction mixture was transferred to a Kontes reaction vessel. Hexane (4 $\mathrm{mL}$ ) was then added, causing the formation of a yellow precipitate. The vessel was sealed with a Teflon valve and immersed in a $135^{\circ} \mathrm{C}$ oil bath. After a few minutes, most of the yellow solid had dissolved and the solution had developed a red color. After heating for ca. $24 \mathrm{~h}$ the reaction vessel was allowed to cool and was transferred to a glove box. The supernatant was removed by pipette and the crystalline product was washed with $\mathrm{Et}_{2} \mathrm{O}(2 \times 2 \mathrm{~mL})$ then pentane $(2 \times 2 \mathrm{~mL})$. Material collected from the reaction mixture was analytically pure (43 $\mathrm{mg}, 0.018 \mathrm{mmol}, 57 \%)$.

$\left(p-M_{e C} \mathbf{H}_{4} \mathbf{N}\right)_{14} \mathbf{T a}_{6} \mathbf{O}$ (3), from compound 1. Compound 1 (100 mg, $0.190 \mathrm{mmol}$, 1 equiv) was dissolved in $1 \mathrm{~mL}$ of benzene. $p$-Toluidine $(102 \mathrm{mg}, 0.950 \mathrm{mmol}, 5$ equiv) was treated with $0.6 \mu \mathrm{L}(0.03$ mmol, 0.16 equiv) of degassed water via microsyringe and the resulting mixture was dissolved in 0.5 $\mathrm{mL}$ benzene. The two solutions were mixed, and the reaction mixture was transferred to a Kontes reaction vessel. Hexane $(4 \mathrm{~mL})$ was then added, causing the formation of a yellow precipitate. The 
vessel was sealed with a Teflon valve and immersed in a $135^{\circ} \mathrm{C}$ oil bath. After a few minutes, most of the yellow solid had dissolved and the solution had developed a red color. After heating for ca. $24 \mathrm{~h}$ the reaction vessel was allowed to cool and was transferred to a glove box. The supernatant was removed by pipette and the crystalline product was washed with $\mathrm{Et}_{2} \mathrm{O}(2 \times 2 \mathrm{~mL})$ then pentane $(2 \times 2 \mathrm{~mL})$. Isotopically-enriched samples were prepared in an identical fashion using ${ }^{17} \mathrm{O} /{ }^{18} \mathrm{O}$ enriched water. Material collected from the reaction mixture was analytically pure $(39.2 \mathrm{mg}, 0.015 \mathrm{mmol}, 48 \%)$.

Reaction conditions from which crystals of 7 were obtained. Compound 1 (10.0 mg, 0.019 mmol, 1 equiv) was dissolved in $0.6 \mathrm{~mL}$ of $\mathrm{C}_{6} \mathrm{D}_{6}$, and the solution was added to $13.5 \mathrm{mg}(0.106 \mathrm{mmol}, 5.56$ equiv) of $m$-chloroaniline. The reaction mixture was then transferred to an NMR tube that contained trace tantalum oxide from previous experiments. The NMR tube was flame sealed under reduced pressure and heated at $135^{\circ} \mathrm{C}$ in an oil bath for $24 \mathrm{~h}$. The reaction mixture was then allowed to stand at ambient temperature for ca. three weeks during which time red/orange crystals of 7 formed.

Ta(OSiPh $)_{5}$ (12). Compound 3 (50 mg, $0.019 \mathrm{mmol}, 1.0$ equiv) was dissolved in $5 \mathrm{~mL}$ of benzene and $156 \mathrm{mg}$ (0.564 mmol, 29.0 equiv) of triphenylsilanol were added. The reaction mixture became yellow-orange in color after being stirred for about $1 \mathrm{~h}$. After $2 \mathrm{~d}$, the light yellow solution was cooled to freezing and the solvent was removed under reduced pressure. The resulting amorphous residue was washed with $\mathrm{Et}_{2} \mathrm{O}(3 \times 1 \mathrm{~mL})$, leaving a white powder which was then dissolved with heating in $1 \mathrm{~mL}$ of benzene. Pentane vapor $(1 \mathrm{~mL}$ total) was allowed to diffuse into the benzene solution over $3 \mathrm{~d}$. The supernatant was then decanted and the crystalline $12 \cdot \mathrm{C}_{6} \mathrm{H}_{6}(103 \mathrm{mg}, 0.0629 \mathrm{mmol}, 54 \%)$ was washed with $\mathrm{Et}_{2} \mathrm{O}(2 \times 1 \mathrm{~mL})$. Some of the crystals were suitable for analysis by X-ray diffraction. ${ }^{1} \mathrm{H}$ NMR $\left(500 \mathrm{MHz}, \mathrm{CD}_{2} \mathrm{Cl}_{2}\right): \delta 7.16(\mathrm{~m}, 45 \mathrm{H}), 6.82(\mathrm{~d}, J=7.5 \mathrm{~Hz}, 30 \mathrm{H}) .{ }^{13} \mathrm{C}\left\{{ }^{1} \mathrm{H}\right\} \mathrm{NMR}\left(125.8 \mathrm{MHz}, \mathrm{CD}_{2} \mathrm{Cl}_{2}\right)$ : $\delta$ 135.87, 129.77, 128.86, 127.96. Anal. Calcd. for $\mathrm{C}_{90} \mathrm{H}_{75} \mathrm{O}_{5} \mathrm{Si}_{5} \mathrm{Ta}_{2} \mathrm{C}_{6} \mathrm{H}_{6}: \mathrm{C}, 70.48 ; \mathrm{H}, 4.99$. Found: C, $70.38 ; \mathrm{H}, 4.67$.

$\mathbf{C} \mathbf{p}^{*} \mathbf{C l}_{2} \mathbf{R e}=\mathbf{N}-\mathbf{p}-\mathbf{C}_{6} \mathbf{H}_{4} \mathbf{M e}$ (13). Compound 3 (100 mg, $0.0388 \mathrm{mmol}, 1.00$ equiv) was dissolved in 10 $\mathrm{mL}$ of benzene and $223 \mathrm{mg}\left(0.546 \mathrm{mmol}, 14.1\right.$ equiv) of $\mathrm{Cp}^{*} \mathrm{Cl}_{2} \mathrm{Re}=\mathrm{O}$ was added. The mixture was 
transferred to a Pyrex reaction vessel fitted with a vacuum stopcock and heated at $130{ }^{\circ} \mathrm{C}$ for $24 \mathrm{~h}$, during which time the solution became deep green in color and a black precipitate formed. NMR tube experiments showed reaction at $75^{\circ} \mathrm{C}$ but longer reaction times were necessary at that temperature. Upon cooling, crystalline 13 precipitated. The solvent was removed under vacuum, and the residue was extracted with $15 \mathrm{~mL}$ of hot THF. After the extract had cooled to ambient temperature, it was cooled at $30{ }^{\circ} \mathrm{C}$ overnight. The supernatant was then removed by pipette and the large green crystals of 13.0.5THF (176 mg, $0.330 \mathrm{mmol}, 61 \%)$ were washed with $\mathrm{Et}_{2} \mathrm{O}(3 \times 1 \mathrm{~mL})$. Some of these crystals were suitable for X-ray diffraction analysis. Note: One half of a molecule of THF was located in the asymmetric unit and thus this formulation was used to calculate the yield and elemental analysis. NMR spectra showed that bulk sample only contained 0.43 equivalents of THF per molecule of $13 .{ }^{1} \mathrm{H}$ NMR $\left(500 \mathrm{MHz}, \mathrm{CD}_{2} \mathrm{Cl}_{2}\right): \delta 7.46(\mathrm{~d}, J=8.5 \mathrm{~Hz}, 2 \mathrm{H}), 7.12(\mathrm{~d}, J=8.5 \mathrm{~Hz}, 2 \mathrm{H}), 2.250(\mathrm{~s}, 3 \mathrm{H}), 2.095(\mathrm{~s}, 15 \mathrm{H})$. ${ }^{13} \mathrm{C}\left\{{ }^{1} \mathrm{H}\right\}$ NMR $\left(125.8 \mathrm{MHz}, \mathrm{CD}_{2} \mathrm{Cl}_{2}\right): \delta 155.21,140.07,130.75,123.67,109.03,22.30,12.64$. Anal. Calcd. for $\mathrm{C}_{17} \mathrm{H}_{22} \mathrm{Cl}_{2} \mathrm{NRe}$ : C, 41.04; H, 4.46; N, 2.81. Anal. Calcd. for $\mathrm{C}_{17} \mathrm{H}_{22} \mathrm{Cl}_{2} \mathrm{NRe} \cdot 0.5 \mathrm{C}_{4} \mathrm{H}_{8} \mathrm{O}$ : C, 42.77; H, 4.91; N, 2.62. Found: C, 42.19; H, 4.66; N, 2.96.

$\mathbf{C p}^{*} \mathbf{C l}_{2} \mathbf{R e}=\mathbf{N}-\boldsymbol{p}-\mathbf{C}_{6} \mathbf{H}_{4}-\mathbf{t}-\mathbf{B u}$ (14). Compound 5 (10 mg, $0.0032 \mathrm{mmol}, 1.0$ equiv) was dissolved in 0.5 $\mathrm{mL}$ of THF- $d_{8}$, and the solution was added to a vial containing $18 \mathrm{mg}(0.044 \mathrm{mmol}, 14$ equiv) of $\mathrm{Cp}^{*} \mathrm{Cl}_{2} \mathrm{Re}=\mathrm{O}$. The reaction mixture was then transferred to an NMR tube and flame sealed under reduced pressure. The NMR tube was heated and the reaction was periodically monitored by ${ }^{1} \mathrm{H}$ NMR. Very little conversion was observed after $7 \mathrm{~h}$ at $75^{\circ} \mathrm{C}$; however, signals assigned to 14 were the only observed resonances after the reaction mixture was heated for $14 \mathrm{~h}$ at $135{ }^{\circ} \mathrm{C}$. The reaction mixture was filtered and the solvent was removed under reduced pressure $(18 \mathrm{mg}, 0.033 \mathrm{mmol}, 75 \%) .{ }^{1} \mathrm{H}$ NMR $(500$ $\left.\mathrm{MHz}, \mathrm{CD}_{2} \mathrm{Cl}_{2}\right): \delta 7.50(\mathrm{~d}, J=9.0 \mathrm{~Hz}, 2 \mathrm{H}), 7.33(\mathrm{~d}, J=9.0 \mathrm{~Hz}, 2 \mathrm{H}), 2.11(\mathrm{~s}, 15 \mathrm{H}), 1.31(\mathrm{~s}, 9 \mathrm{H}) .{ }^{13} \mathrm{C}$ $\left\{{ }^{1} \mathrm{H}\right\}$ NMR $\left(125.8 \mathrm{MHz}, \mathrm{CD}_{2} \mathrm{Cl}_{2}\right): \delta 155.08,152.96,127.15,123.44,109.06,35.88,31.04,12.69$. Anal. Calcd. for $\mathrm{C}_{20} \mathrm{H}_{28} \mathrm{Cl}_{2} \mathrm{NRe}$ : C, 44.52; H, 5.23; N, 2.60. Found: C, 44.23; H, 5.12; N, 2.88 . 


\section{Electrospray Mass Spectra for 3 and 4, Including ${ }^{17} \mathrm{O}-,{ }^{18} \mathrm{O}$-Enriched Samples}

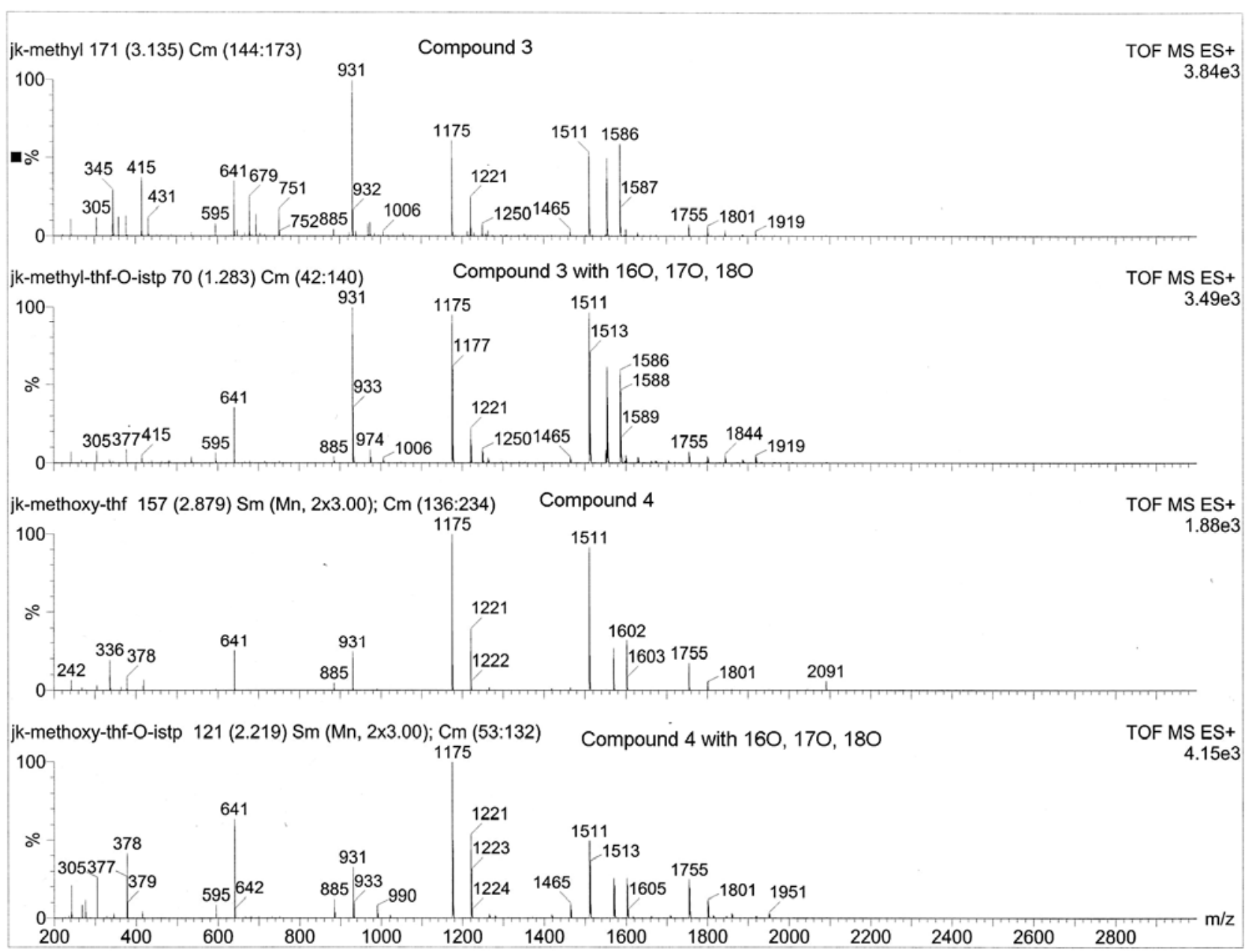

Figure S1. ESI masss spectra of 3, 3( $\left({ }^{17,18} \mathrm{O}\right), \mathbf{4}$, and $\mathbf{4}\left({ }^{17,18} \mathrm{O}\right)$ (from top down, respectively). Full spectral width. 


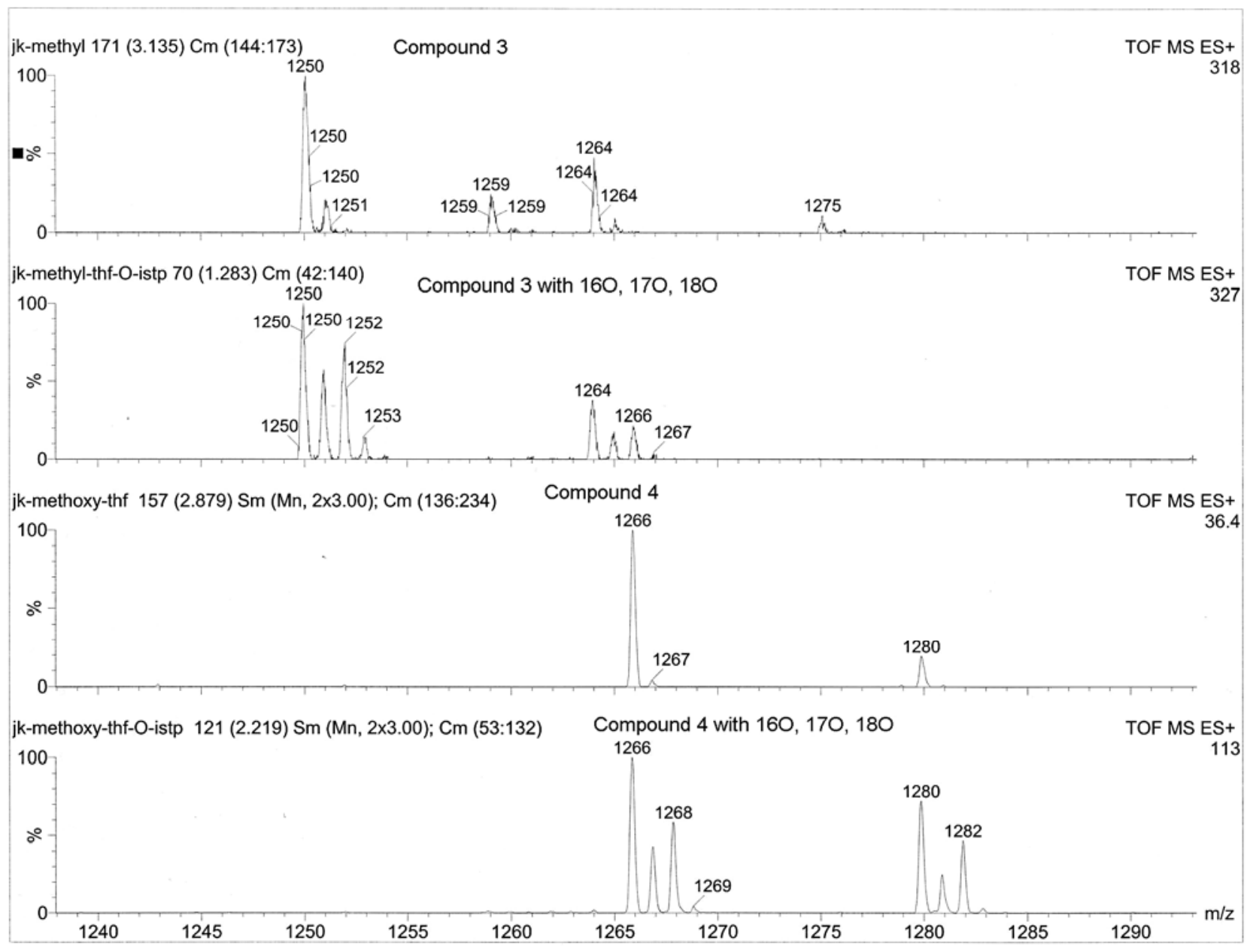

Figure S2. Enlargement \#1, showing 16 Da shifts of compound 4 peaks relative to those of 3. 


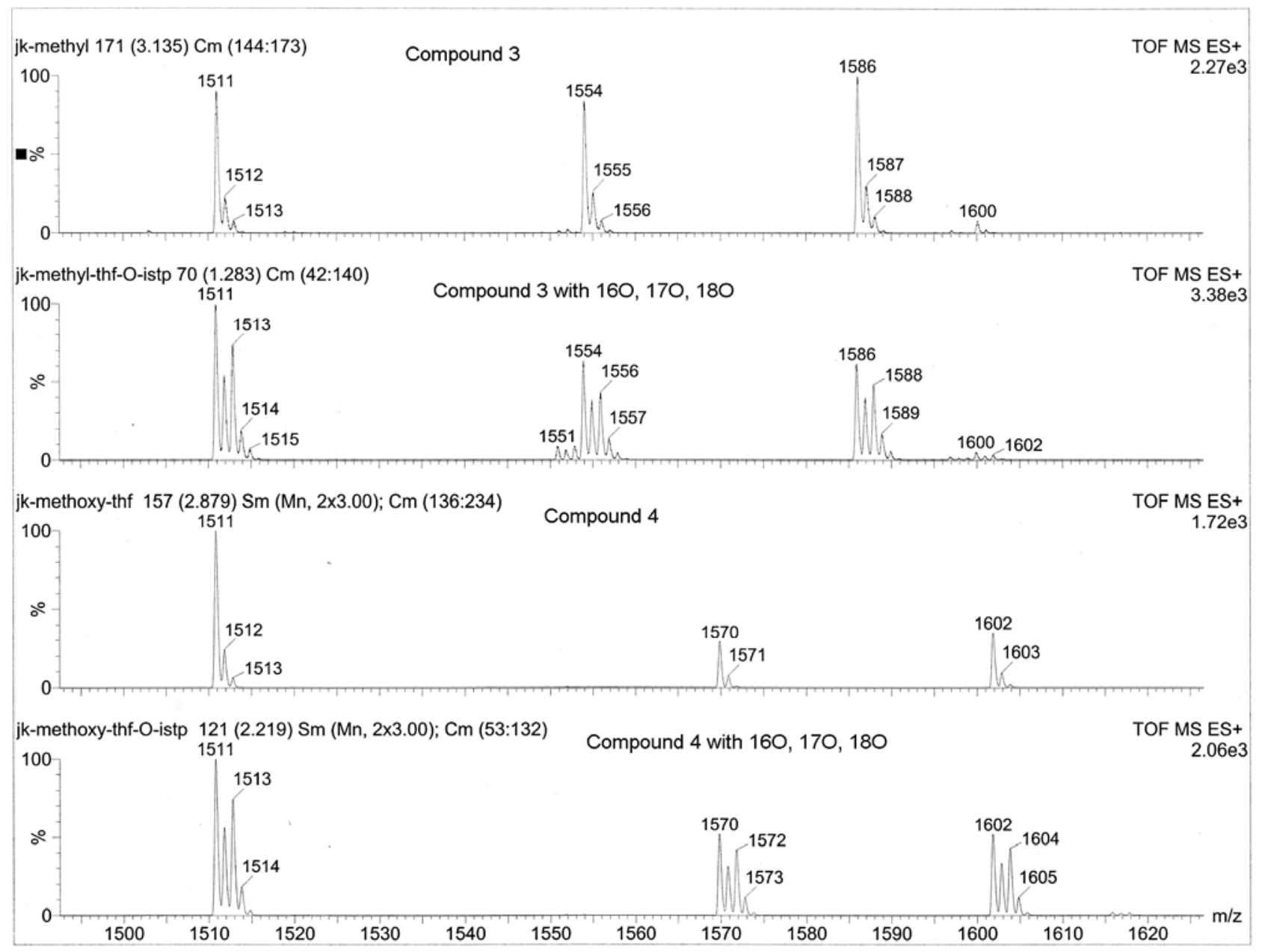

Figure S3. Enlargement $\# 2$, showing peak that is common to $\mathbf{3}$ and $\mathbf{4}$, no ligands remaining on cluster core. 


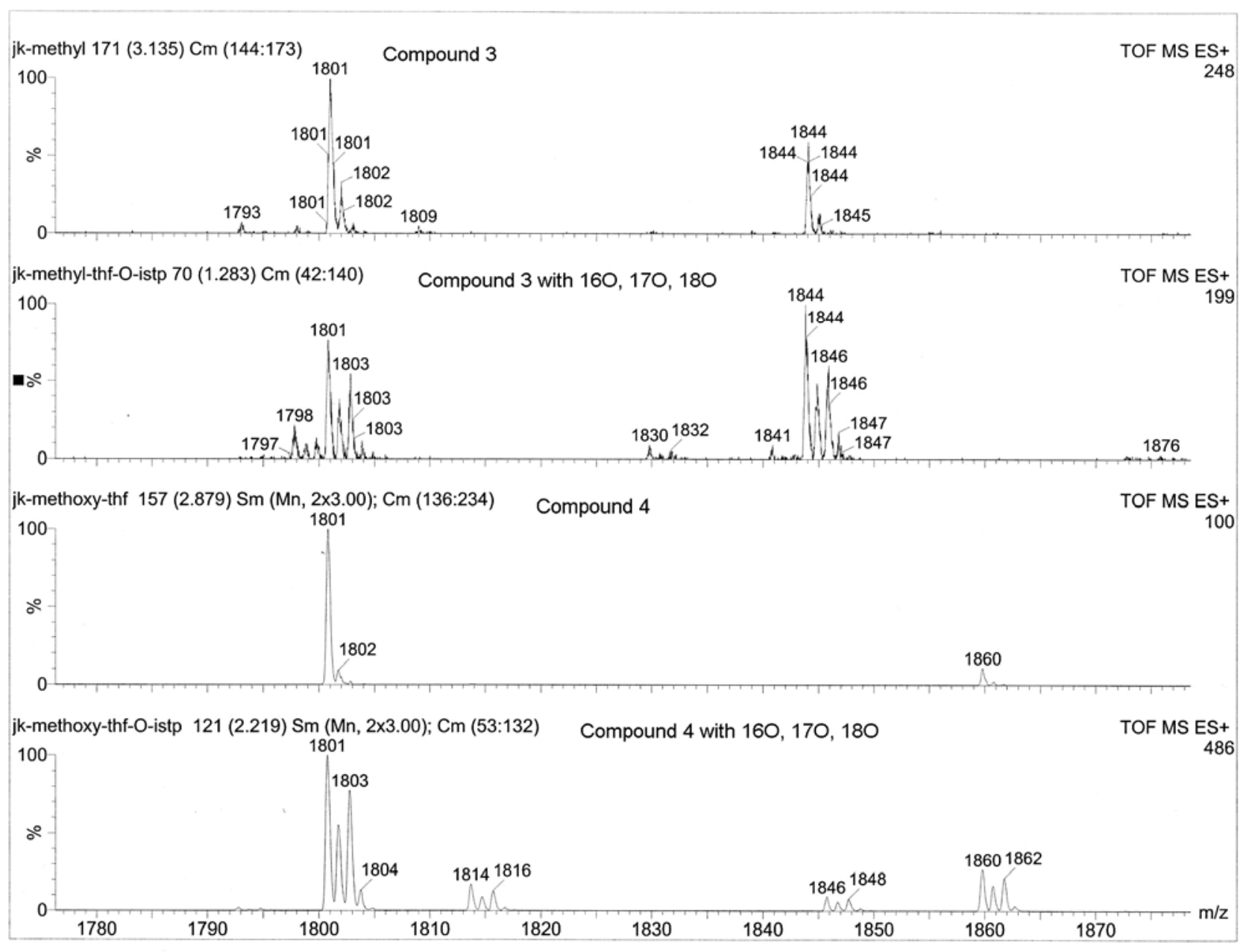

Figure S4. Enlargement \#3, showing higher-mass peaks.

\section{Positional coordinates of computed structures.}

Table S1. Calculated coordinates for optimized 2, unconstrained.

$\begin{array}{llll}\text { Ta1 } & 1.2806743833 & -0.8537378180 & 1.5971574106 \\ \text { Ta2 } & 0.1572970891 & 2.0112842388 & 0.9697664333 \\ \text { Ta3 } & -1.8100981746 & -0.4348225289 & 1.1928484134 \\ \text { N4 } & -0.2459704993 & 0.4426922028 & 2.4684537392 \\ \text { N5 } & 2.1322883908 & 1.0258682528 & 0.9017255418 \\ \text { N6 } & 2.2963615069 & -1.5854991031 & 2.8718160051 \\ \text { N7 } & 0.2779376663 & 3.6086951996 & 1.7607308190 \\ \text { N8 } & 0.4720756545 & 2.1820154373 & -1.1977352550 \\ \text { N9 } & -1.8999774085 & 1.6155086839 & 0.3754197685 \\ \text { N10 } & -3.2759591765 & -0.7994299133 & 2.1503842702 \\ \text { O11 } & 0.0047097086 & 0.0131439042 & -0.0039601891 \\ \text { C12 } & -0.3715422756 & 0.5462608774 & 3.8923886073 \\ \text { C13 } & -0.1036635726 & 1.7391893568 & 4.5796361817 \\ \text { H14 } & 0.1958538477 & 2.6207525502 & 4.0242482342 \\ \text { C15 } & -0.2215297416 & 1.7979643395 & 5.9687029083\end{array}$




\begin{tabular}{|c|c|c|c|}
\hline H16 & -0.0092860434 & 2.7349305122 & 6.4771152386 \\
\hline C17 & -0.6026969401 & 0.6737025869 & 6.7019867278 \\
\hline H18 & -0.6909100408 & 0.7244392244 & 7.7837884831 \\
\hline C19 & -0.8667262502 & -0.5185744253 & 6.0259882814 \\
\hline $\mathrm{H} 20$ & -1.1612113902 & -1.4082868954 & 6.5768559207 \\
\hline $\mathrm{C} 21$ & -0.7537674555 & -0.5834512471 & 4.6392821199 \\
\hline $\mathrm{H} 22$ & -0.9573369144 & -1.5201158017 & 4.1278861355 \\
\hline $\mathrm{C} 23$ & 3.2953218147 & 1.6978295268 & 1.3981479109 \\
\hline $\mathrm{C} 24$ & 3.8275348826 & 1.4091295638 & 2.6647411592 \\
\hline $\mathrm{H} 25$ & 3.3566410629 & 0.6545721500 & 3.2851788691 \\
\hline $\mathrm{C} 26$ & 4.9600439590 & 2.0773064419 & 3.1274416764 \\
\hline $\mathrm{H} 27$ & 5.3530276217 & 1.8317890590 & 4.1106900731 \\
\hline $\mathrm{C} 28$ & 5.5855391416 & 3.0469123757 & 2.3442285083 \\
\hline H29 & 6.4699321639 & 3.5624270710 & 2.7080288185 \\
\hline $\mathrm{C} 30$ & 5.0583213812 & 3.3472598441 & 1.0876952903 \\
\hline H31 & 5.5282747224 & 4.1019720542 & 0.4625765007 \\
\hline C32 & 3.9255116725 & 2.6851422753 & 0.6203330722 \\
\hline H33 & 3.5253327472 & 2.9298151442 & -0.3586589119 \\
\hline C34 & 3.0635336594 & -2.1902246561 & 3.8451181281 \\
\hline $\mathrm{C} 35$ & 4.0509835853 & -3.1278249887 & 3.4899718331 \\
\hline H36 & 4.2095927902 & -3.3534383514 & 2.4396899502 \\
\hline C37 & 4.8158552086 & -3.7416295927 & 4.4772949127 \\
\hline H38 & 5.5757743384 & -4.4647215089 & 4.1916118154 \\
\hline C39 & 4.6140501205 & -3.4340901375 & 5.8242182655 \\
\hline $\mathrm{H} 40$ & 5.2141171556 & -3.9167714162 & 6.5909221821 \\
\hline C41 & 3.6363444193 & -2.5029149198 & 6.1799438918 \\
\hline H42 & 3.4719301122 & -2.2593809537 & 7.2265762648 \\
\hline $\mathrm{C} 43$ & 2.8626420560 & -1.8817657770 & 5.2033906085 \\
\hline H44 & 2.0901390833 & -1.1670148122 & 5.4719095530 \\
\hline $\mathrm{C} 45$ & 0.3628083449 & 4.8364732702 & 2.3839802365 \\
\hline C46 & 1.6119939554 & 5.3343539625 & 2.7994707005 \\
\hline H47 & 2.5032107183 & 4.7416629677 & 2.6122847910 \\
\hline C48 & 1.6908588663 & 6.5696812311 & 3.4357003348 \\
\hline H49 & 2.6597022848 & 6.9459407991 & 3.7541524162 \\
\hline $\mathrm{C} 50$ & 0.5381434493 & 7.3230312643 & 3.6658239126 \\
\hline H51 & 0.6061444386 & 8.2862762339 & 4.1642693428 \\
\hline C52 & -0.7024896965 & 6.8322211183 & 3.2531400665 \\
\hline H53 & -1.6040007162 & 7.4134363182 & 3.4296249416 \\
\hline C54 & -0.7979457927 & 5.5984674946 & 2.6157389536 \\
\hline H55 & -1.7587733726 & 5.2047443332 & 2.2963819269 \\
\hline C56 & 0.6766234825 & 3.3600946347 & -1.9838291286 \\
\hline C57 & 1.0040544702 & 3.2497691366 & -3.3478545451 \\
\hline H58 & 1.1110873328 & 2.2658611328 & -3.7958446104 \\
\hline C59 & 1.1831253971 & 4.3871698623 & -4.1318158827 \\
\hline H60 & 1.4313650739 & 4.2727454495 & -5.1838264297 \\
\hline C61 & 1.0427285747 & 5.6602216496 & -3.5757560562 \\
\hline H62 & 1.1824549739 & 6.5469554691 & -4.1877502392 \\
\hline C63 & 0.7177796879 & 5.7775560538 & -2.2246017181 \\
\hline H64 & 0.6023039151 & 6.7597776783 & -1.7734669623 \\
\hline C65 & 0.5337894373 & 4.6431367073 & -1.4343870201 \\
\hline H66 & 0.2788424737 & 4.7504524551 & -0.3858201228 \\
\hline
\end{tabular}




\begin{tabular}{|c|c|c|c|}
\hline C67 & -2.9337707458 & 2.5720098040 & 0.6240569355 \\
\hline C68 & -3.6184841225 & 2.5984271410 & 1.8513932926 \\
\hline H69 & -3.3684090532 & 1.8758075539 & 2.6205240307 \\
\hline $\mathrm{C} 70$ & -4.6205213021 & 3.5365331451 & 2.0884584905 \\
\hline H71 & -5.1363797712 & 3.5305740569 & 3.0452107282 \\
\hline $\mathrm{C} 72$ & -4.9597620523 & 4.4733919312 & 1.1111934319 \\
\hline H73 & -5.7429531504 & 5.2033643942 & 1.2959777160 \\
\hline C74 & -4.2776754492 & 4.4600882846 & -0.1057686037 \\
\hline H75 & -4.5242127023 & 5.1830469490 & -0.8789950213 \\
\hline C76 & -3.2744210352 & 3.5231726866 & -0.3491789246 \\
\hline H77 & -2.7562341096 & 3.5243622915 & -1.3032267446 \\
\hline $\mathrm{C} 78$ & -4.3960045950 & -1.0864358024 & 2.9032978559 \\
\hline C79 & -4.4849277867 & -0.6518645544 & 4.2396572743 \\
\hline H80 & -3.6560114358 & -0.0975635074 & 4.6691504969 \\
\hline C81 & -5.6133399760 & -0.9499022367 & 4.9981658665 \\
\hline H82 & -5.6689511583 & -0.6104992271 & 6.0295442267 \\
\hline C83 & -6.6661393076 & -1.6812520855 & 4.4451418375 \\
\hline H84 & -7.5441904735 & -1.9128497292 & 5.0420295344 \\
\hline $\mathrm{C} 85$ & -6.5827200436 & -2.1139024227 & 3.1205001511 \\
\hline H86 & -7.3987254494 & -2.6825715838 & 2.6816114802 \\
\hline C87 & -5.4608352121 & -1.8220415880 & 2.3497100265 \\
\hline H88 & -5.3965722317 & -2.1441045717 & 1.3147606515 \\
\hline Ta89 & -1.2715442143 & 0.8839694287 & -1.6025761335 \\
\hline Ta90 & -0.1443228855 & -1.9887385192 & -0.9685264689 \\
\hline Ta91 & 1.8123771318 & 0.4567723414 & -1.2045765289 \\
\hline N92 & 0.2424450042 & -0.4284393375 & -2.4768448266 \\
\hline N93 & -2.1289135483 & -0.9944216154 & -0.8931372313 \\
\hline N94 & -2.3034713854 & 1.5953122157 & -2.8746438851 \\
\hline N95 & -0.2794642053 & -3.6023866744 & -1.7246974866 \\
\hline N96 & -0.4584365595 & -2.1581735754 & 1.1831116860 \\
\hline N97 & 1.9165318915 & -1.5846597216 & -0.3844573947 \\
\hline N98 & 3.2713527105 & 0.7979393182 & -2.1782941016 \\
\hline C99 & 0.3580492545 & -0.7776678697 & -3.8606834990 \\
\hline $\mathrm{C} 100$ & -0.7699727477 & -0.7906105824 & -4.6982341219 \\
\hline H101 & -1.7401955903 & -0.5177169910 & -4.2955928216 \\
\hline $\mathrm{C} 102$ & -0.6577468568 & -1.1417634149 & -6.0424528023 \\
\hline H103 & -1.5495994324 & -1.1383180112 & -6.6638602696 \\
\hline C104 & 0.5780966789 & -1.4934859070 & -6.5877963982 \\
\hline H105 & 0.6621045384 & -1.7654073375 & -7.6359639944 \\
\hline C106 & 1.7034473283 & -1.4880847321 & -5.7618893432 \\
\hline H107 & 2.6770820895 & -1.7585454337 & -6.1622061792 \\
\hline $\mathrm{C} 108$ & 1.5976756913 & -1.1389386950 & -4.4160739388 \\
\hline H109 & 2.4842452257 & -1.1402252638 & -3.7905097377 \\
\hline $\mathrm{C} 110$ & -3.3965108196 & -1.4632677610 & -1.3656139175 \\
\hline C111 & -4.5492006092 & -0.6827858421 & -1.1591789693 \\
\hline H112 & -4.4659546867 & 0.2701838626 & -0.6441095867 \\
\hline C113 & -5.7945218503 & -1.1185061194 & -1.6057696324 \\
\hline H114 & -6.6689100147 & -0.4971078484 & -1.4294929442 \\
\hline $\mathrm{C} 115$ & -5.9201754036 & -2.3402933075 & -2.2700827153 \\
\hline H116 & -6.8918266216 & -2.6809659407 & -2.6169521212 \\
\hline C117 & -4.7811561644 & -3.1164636455 & -2.4821601287 \\
\hline
\end{tabular}




\begin{tabular}{|c|c|c|c|}
\hline H118 & -4.8589066097 & -4.0700813312 & -2.9981301695 \\
\hline C119 & -3.5311053760 & -2.6864684175 & -2.0374536850 \\
\hline H120 & -2.6561730361 & -3.3027864571 & -2.2084569176 \\
\hline C121 & -3.1127120915 & 2.1603205171 & -3.8383171991 \\
\hline $\mathrm{C} 122$ & -4.2306867997 & 1.4626313910 & -4.3315071632 \\
\hline H123 & -4.4506090807 & 0.4749658496 & -3.9374802636 \\
\hline C124 & -5.0459665602 & 2.0465006499 & -5.2968596809 \\
\hline H125 & -5.9087501507 & 1.4994806695 & -5.6689113970 \\
\hline C126 & -4.7651448832 & 3.3249054455 & -5.7832603070 \\
\hline H127 & -5.4066409944 & 3.7770619217 & -6.5349110811 \\
\hline C128 & -3.6551839909 & 4.0187856738 & -5.2971334515 \\
\hline H129 & -3.4286622783 & 5.0140666838 & -5.6712167376 \\
\hline C130 & -2.8305724187 & 3.4470438253 & -4.3324895590 \\
\hline H131 & -1.9615936360 & 3.9774243288 & -3.9534318327 \\
\hline C132 & -0.4135815931 & -4.8484876692 & -2.3052684243 \\
\hline C133 & -0.7777745486 & -5.9663248822 & -1.5283699623 \\
\hline H134 & -0.9481583469 & -5.8333487956 & -0.4637750357 \\
\hline C135 & -0.9212822379 & -7.2138722055 & -2.1292654490 \\
\hline H136 & -1.2043431570 & -8.0706338823 & -1.5229750568 \\
\hline C137 & -0.7069117347 & -7.3677652863 & -3.5005180876 \\
\hline H138 & -0.8222836833 & -8.3431403410 & -3.9656960213 \\
\hline C139 & -0.3446162506 & -6.2618236973 & -4.2716221354 \\
\hline H140 & -0.1759996981 & -6.3751742228 & -5.3394781753 \\
\hline C141 & -0.1970752290 & -5.0075363491 & -3.6860015705 \\
\hline H142 & 0.0829289780 & -4.1409238903 & -4.2782895006 \\
\hline C143 & -0.6985120285 & -3.4313582629 & 1.7913774833 \\
\hline C144 & -2.0070773612 & -3.9107581865 & 1.9712840039 \\
\hline H145 & -2.8480739731 & -3.3043881322 & 1.6520432691 \\
\hline C146 & -2.2376314140 & -5.1509594939 & 2.5634053526 \\
\hline H147 & -3.2610549989 & -5.4931357048 & 2.6939475569 \\
\hline C148 & -1.1715533937 & -5.9462135529 & 2.9842326695 \\
\hline H149 & -1.3534136447 & -6.9118509016 & 3.4478161382 \\
\hline $\mathrm{C} 150$ & 0.1317312570 & -5.4835753079 & 2.8000672323 \\
\hline H151 & 0.9774162232 & -6.0876939291 & 3.1184081234 \\
\hline C152 & 0.3686163732 & -4.2439408892 & 2.2083144156 \\
\hline H153 & 1.3889131146 & -3.8999411922 & 2.0765673958 \\
\hline C154 & 3.0835101270 & -2.4033214644 & -0.5199639341 \\
\hline $\mathrm{C} 155$ & 3.0127185060 & -3.7181467277 & -1.0042551535 \\
\hline H156 & 2.0526364281 & -4.1287022057 & -1.2945247340 \\
\hline C157 & 4.1654271855 & -4.4946427967 & -1.1170134962 \\
\hline H158 & 4.0830996764 & -5.5098152514 & -1.4968337874 \\
\hline C159 & 5.4097600620 & -3.9822360760 & -0.7495486743 \\
\hline H160 & 6.3047438414 & -4.5916955116 & -0.8389240919 \\
\hline C161 & 5.4894161829 & -2.6747889161 & -0.2658353626 \\
\hline H162 & 6.4486575672 & -2.2551792804 & 0.0263269156 \\
\hline C163 & 4.3421422205 & -1.8926525574 & -0.1520379946 \\
\hline H164 & 4.4197087022 & -0.8777944305 & 0.2280438241 \\
\hline $\mathrm{C} 165$ & 4.3884653931 & 1.0371412418 & -2.9497263911 \\
\hline C166 & 5.2555743313 & -0.0188063774 & -3.2882590909 \\
\hline H167 & 5.0448569777 & -1.0153310046 & -2.9115438420 \\
\hline C168 & 6.3719121770 & 0.2227262614 & -4.0837501551 \\
\hline
\end{tabular}




$\begin{array}{llll}\text { H169 } & 7.0334885579 & -0.6011934980 & -4.3393034838 \\ \text { C170 } & 6.6450961803 & 1.5097476016 & -4.5505016377 \\ \text { H171 } & 7.5169369639 & 1.6924980301 & -5.1728346597 \\ \text { C172 } & 5.7905842063 & 2.5608059050 & -4.2126341942 \\ \text { H173 } & 5.9958336606 & 3.5662134397 & -4.5715383172 \\ \text { C174 } & 4.6702537627 & 2.3337093013 & -3.4189100750 \\ \text { H175 } & 3.9942482895 & 3.1441972493 & -3.1631001794\end{array}$

Table S2. Calculated coordinates for $C_{i}$-optimized 2.

$\begin{array}{llll}\text { Ta1 } & 1.2792696352 & -0.9206652122 & 1.5736753355 \\ \text { Ta2 } & 0.2060455097 & 1.9833567331 & 0.9806115666 \\ \text { Ta3 } & -1.8088255836 & -0.4273567693 & 1.2170053725 \\ \text { N4 } & -0.2040941221 & 0.3947175746 & 2.4764755010 \\ \text { N5 } & 2.1754757491 & 0.9094017597 & 0.9059241824 \\ \text { N6 } & 2.2907007824 & -1.7218594681 & 2.8117794926 \\ \text { N7 } & 0.4349557612 & 3.5725637237 & 1.7644629300 \\ \text { N8 } & 0.5451980369 & 2.1728011567 & -1.1670576029 \\ \text { N9 } & -1.8508676926 & 1.6652733032 & 0.4382430588 \\ \text { N10 } & -3.2941999681 & -0.7575413702 & 2.1549412685 \\ \text { O11 } & 0.0000000000 & 0.0000000000 & 0.0000000000 \\ \text { C12 } & -0.3062871444 & 0.7738951731 & 3.8509594451 \\ \text { C13 } & 0.7982117100 & 0.6975878133 & 4.7146509825 \\ \text { H14 } & 1.7487380385 & 0.3329557894 & 4.3380206048 \\ \text { C15 } & 0.6861467758 & 1.0856675521 & 6.0489282211 \\ \text { H16 } & 1.5565348238 & 1.0105929950 & 6.6964596374 \\ \text { C17 } & -0.5230054332 & 1.5674451770 & 6.5513229655 \\ \text { H18 } & -0.6061463528 & 1.8692141749 & 7.5919932134 \\ \text { C19 } & -1.6216129163 & 1.6626984342 & 5.6962225772 \\ \text { H20 } & -2.5705871049 & 2.0455939134 & 6.0638198440 \\ \text { C21 } & -1.5163698765 & 1.2737750666 & 4.3625989213 \\ \text { H22 } & -2.3787306584 & 1.3578790516 & 3.7077467258 \\ \text { C23 } & 3.3839518773 & 1.5489229644 & 1.3310008455 \\ \text { C24 } & 4.3019630879 & 0.9132659800 & 2.1815829369 \\ \text { H25 } & 4.0988800787 & -0.0929828892 & 2.5309936314 \\ \text { C26 } & 5.4657948124 & 1.5674003389 & 2.5856698607 \\ \text { H27 } & 6.1593325682 & 1.0528327128 & 3.2466535929 \\ \text { C28 } & 5.7402394683 & 2.8654008537 & 2.1567583228 \\ \text { H29 } & 6.6460959722 & 3.3727311947 & 2.4778794453 \\ \text { C30 } & 4.8338990441 & 3.5045216495 & 1.3094739761 \\ \text { H31 } & 5.0262588954 & 4.5170890200 & 0.9635752055 \\ \text { C32 } & 3.6726100149 & 2.8559801355 & 0.8989428824 \\ \text { H33 } & 2.9783068384 & 3.3690053734 & 0.2399955124 \\ \text { C34 } & 3.0569554648 & -2.3803015742 & 3.7516277150 \\ \text { C35 } & 4.2951920193 & -2.9444952228 & 3.3910477652 \\ \text { H36 } & 4.6282923767 & -2.8686315786 & 2.3596113537 \\ \text { C37 } & 5.0700778343 & -3.5951596980 & 4.3472031878 \\ \text { H38 } & 6.0246605740 & -4.0275181075 & 4.0564825047 \\ \text { C39 } & 4.6311908977 & -3.6947605645 & 5.6685268003 \\ \text { C41 } & 5.2411757176 & -4.2031751518 & 6.4107669471 \\ & 3.4021638230 & -3.1391745765 & 6.0298891889\end{array}$




\begin{tabular}{|c|c|c|c|}
\hline H42 & 3.0510158658 & -3.2145534868 & 7.0562429247 \\
\hline $\mathrm{C} 43$ & 2.6168210874 & -2.4863278021 & 5.0845978931 \\
\hline H44 & 1.6605423803 & -2.0499206476 & 5.3578098065 \\
\hline $\mathrm{C} 45$ & 0.6491723913 & 4.7863049571 & 2.3834555104 \\
\hline $\mathrm{C} 46$ & 0.7431947644 & 5.9651546566 & 1.6200170167 \\
\hline H47 & 0.6490699902 & 5.9004242857 & 0.5391929943 \\
\hline $\mathrm{C} 48$ & 0.9506041688 & 7.1895060768 & 2.2496777296 \\
\hline H49 & 1.0231660679 & 8.0935833490 & 1.6494258518 \\
\hline C50 & 1.0663872218 & 7.2599938203 & 3.6386096808 \\
\hline H51 & 1.2261181260 & 8.2185013233 & 4.1258951549 \\
\hline C52 & 0.9751014252 & 6.0918252296 & 4.3989783779 \\
\hline H53 & 1.0652012493 & 6.1393660934 & 5.4815205488 \\
\hline C54 & 0.7690390183 & 4.8609336310 & 3.7837549305 \\
\hline H55 & 0.6948227073 & 3.9473484965 & 4.3672507794 \\
\hline C56 & 0.7186345497 & 3.4399131000 & -1.8123031319 \\
\hline C57 & 1.9254936414 & 3.7924316562 & -2.4346555162 \\
\hline H58 & 2.7548577550 & 3.0947597270 & -2.4250511781 \\
\hline C59 & 2.0609881124 & 5.0244860718 & -3.0733444452 \\
\hline H60 & 3.0063202604 & 5.2710094035 & -3.5507718031 \\
\hline C61 & 1.0008456398 & 5.9289233304 & -3.1103043883 \\
\hline H62 & 1.1087808696 & 6.8852122459 & -3.6151072141 \\
\hline C63 & -0.2034768238 & 5.5872032378 & -2.4929222996 \\
\hline H64 & -1.0449730680 & 6.2754811199 & -2.5129490319 \\
\hline C65 & -0.3439107105 & 4.3607841811 & -1.8482428344 \\
\hline H66 & -1.2889628253 & 4.1095520007 & -1.3749971050 \\
\hline C67 & -2.9902567402 & 2.5058245121 & 0.6455141426 \\
\hline C68 & -2.8627514971 & 3.8296224671 & 1.0935170180 \\
\hline H69 & -1.8764007026 & 4.2320602240 & 1.2961121547 \\
\hline $\mathrm{C} 70$ & -3.9926545593 & 4.6198649153 & 1.3019284241 \\
\hline H71 & -3.8652547684 & 5.6405359319 & 1.6546581610 \\
\hline $\mathrm{C} 72$ & -5.2709152896 & 4.1135557050 & 1.0709336278 \\
\hline H73 & -6.1481109650 & 4.7317385106 & 1.2421074862 \\
\hline C74 & -5.4077939045 & 2.8006042713 & 0.6171758900 \\
\hline H75 & -6.3950464000 & 2.3835907340 & 0.4330563041 \\
\hline C76 & -4.2835951241 & 2.0079780460 & 0.4003004788 \\
\hline H77 & -4.4067179747 & 0.9891556884 & 0.0444977211 \\
\hline $\mathrm{C} 78$ & -4.4656403691 & -0.9997955765 & 2.8411297247 \\
\hline C79 & -4.4849715649 & -0.9895904744 & 4.2481797070 \\
\hline $\mathrm{H} 80$ & -3.5649857758 & -0.7767431022 & 4.7847517288 \\
\hline C81 & -5.6709280059 & -1.2402526938 & 4.9317452725 \\
\hline H82 & -5.6741011075 & -1.2262212582 & 6.0191425861 \\
\hline C83 & -6.8490916988 & -1.5086640692 & 4.2326342127 \\
\hline H84 & -7.7721834314 & -1.7052520677 & 4.7719189874 \\
\hline C85 & -6.8335154522 & -1.5250623723 & 2.8365242301 \\
\hline H86 & -7.7454604736 & -1.7386119635 & 2.2841042195 \\
\hline C87 & -5.6550323808 & -1.2744380750 & 2.1399791165 \\
\hline H88 & -5.6325031731 & -1.2960176711 & 1.0540967970 \\
\hline Ta89 & -1.2792696352 & 0.9206652122 & -1.5736753355 \\
\hline Ta90 & -0.2060455097 & -1.9833567331 & -0.9806115666 \\
\hline Ta91 & 1.8088255836 & 0.4273567693 & -1.2170053725 \\
\hline N92 & 0.2040941221 & -0.3947175746 & -2.4764755010 \\
\hline
\end{tabular}




\begin{tabular}{|c|c|c|c|}
\hline N93 & -2.1754757491 & -0.9094017597 & -0.9059241824 \\
\hline N94 & -2.2907007824 & 1.7218594681 & -2.8117794926 \\
\hline N95 & -0.4349557612 & -3.5725637237 & -1.7644629300 \\
\hline N96 & -0.5451980369 & -2.1728011567 & 1.1670576029 \\
\hline N97 & 1.8508676926 & -1.6652733032 & -0.4382430588 \\
\hline N98 & 3.2941999681 & 0.7575413702 & -2.1549412685 \\
\hline C99 & 0.3062871444 & -0.7738951731 & -3.8509594451 \\
\hline $\mathrm{C} 100$ & -0.7982117100 & -0.6975878133 & -4.7146509825 \\
\hline H101 & -1.7487380385 & -0.3329557894 & -4.3380206048 \\
\hline $\mathrm{C} 102$ & -0.6861467758 & -1.0856675521 & -6.0489282211 \\
\hline H103 & -1.5565348238 & -1.0105929950 & -6.6964596374 \\
\hline C104 & 0.5230054332 & -1.5674451770 & -6.5513229655 \\
\hline H105 & 0.6061463528 & -1.8692141749 & -7.5919932134 \\
\hline C106 & 1.6216129163 & -1.6626984342 & -5.6962225772 \\
\hline H107 & 2.5705871049 & -2.0455939134 & -6.0638198440 \\
\hline $\mathrm{C} 108$ & 1.5163698765 & -1.2737750666 & -4.3625989213 \\
\hline H109 & 2.3787306584 & -1.3578790516 & -3.7077467258 \\
\hline C110 & -3.3839518773 & -1.5489229644 & -1.3310008455 \\
\hline C111 & -4.3019630879 & -0.9132659800 & -2.1815829369 \\
\hline H112 & -4.0988800787 & 0.0929828892 & -2.5309936314 \\
\hline C113 & -5.4657948124 & -1.5674003389 & -2.5856698607 \\
\hline H114 & -6.1593325682 & -1.0528327128 & -3.2466535929 \\
\hline $\mathrm{C} 115$ & -5.7402394683 & -2.8654008537 & -2.1567583228 \\
\hline H116 & -6.6460959722 & -3.3727311947 & -2.4778794453 \\
\hline C117 & -4.8338990441 & -3.5045216495 & -1.3094739761 \\
\hline H118 & -5.0262588954 & -4.5170890200 & -0.9635752055 \\
\hline C119 & -3.6726100149 & -2.8559801355 & -0.8989428824 \\
\hline H120 & -2.9783068384 & -3.3690053734 & -0.2399955124 \\
\hline C121 & -3.0569554648 & 2.3803015742 & -3.7516277150 \\
\hline $\mathrm{C} 122$ & -4.2951920193 & 2.9444952228 & -3.3910477652 \\
\hline H123 & -4.6282923767 & 2.8686315786 & -2.3596113537 \\
\hline C124 & -5.0700778343 & 3.5951596980 & -4.3472031878 \\
\hline H125 & -6.0246605740 & 4.0275181075 & -4.0564825047 \\
\hline C126 & -4.6311908977 & 3.6947605645 & -5.6685268003 \\
\hline H127 & -5.2411757176 & 4.2031751518 & -6.4107669471 \\
\hline C128 & -3.4021638230 & 3.1391745765 & -6.0298891889 \\
\hline H129 & -3.0510158658 & 3.2145534868 & -7.0562429247 \\
\hline $\mathrm{C} 130$ & -2.6168210874 & 2.4863278021 & -5.0845978931 \\
\hline H131 & -1.6605423803 & 2.0499206476 & -5.3578098065 \\
\hline C132 & -0.6491723913 & -4.7863049571 & -2.3834555104 \\
\hline C133 & -0.7431947644 & -5.9651546566 & -1.6200170167 \\
\hline H134 & -0.6490699902 & -5.9004242857 & -0.5391929943 \\
\hline $\mathrm{C} 135$ & -0.9506041688 & -7.1895060768 & -2.2496777296 \\
\hline H136 & -1.0231660679 & -8.0935833490 & -1.6494258518 \\
\hline C137 & -1.0663872218 & -7.2599938203 & -3.6386096808 \\
\hline H138 & -1.2261181260 & -8.2185013233 & -4.1258951549 \\
\hline C139 & -0.9751014252 & -6.0918252296 & -4.3989783779 \\
\hline $\mathrm{H} 140$ & -1.0652012493 & -6.1393660934 & -5.4815205488 \\
\hline C141 & -0.7690390183 & -4.8609336310 & -3.7837549305 \\
\hline H142 & -0.6948227073 & -3.9473484965 & -4.3672507794 \\
\hline C143 & -0.7186345497 & -3.4399131000 & 1.8123031319 \\
\hline
\end{tabular}




$\begin{array}{llll}\text { C144 } & -1.9254936414 & -3.7924316562 & 2.4346555162 \\ \text { H145 } & -2.7548577550 & -3.0947597270 & 2.4250511781 \\ \text { C146 } & -2.0609881124 & -5.0244860718 & 3.0733444452 \\ \text { H147 } & -3.0063202604 & -5.2710094035 & 3.5507718031 \\ \text { C148 } & -1.0008456398 & -5.9289233304 & 3.1103043883 \\ \text { H149 } & -1.1087808696 & -6.8852122459 & 3.6151072141 \\ \text { C150 } & 0.2034768238 & -5.5872032378 & 2.4929222996 \\ \text { H151 } & 1.0449730680 & -6.2754811199 & 2.5129490319 \\ \text { C152 } & 0.3439107105 & -4.3607841811 & 1.8482428344 \\ \text { H153 } & 1.2889628253 & -4.1095520007 & 1.3749971050 \\ \text { C154 } & 2.9902567402 & -2.5058245121 & -0.6455141426 \\ \text { C155 } & 2.8627514971 & -3.8296224671 & -1.0935170180 \\ \text { H156 } & 1.8764007026 & -4.2320602240 & -1.2961121547 \\ \text { C157 } & 3.9926545593 & -4.6198649153 & -1.3019284241 \\ \text { H158 } & 3.8652547684 & -5.6405359319 & -1.6546581610 \\ \text { C159 } & 5.2709152896 & -4.1135557050 & -1.0709336278 \\ \text { H160 } & 6.1481109650 & -4.7317385106 & -1.2421074862 \\ \text { C161 } & 5.4077939045 & -2.8006042713 & -0.6171758900 \\ \text { H162 } & 6.3950464000 & -2.3835907340 & -0.4330563041 \\ \text { C163 } & 4.2835951241 & -2.0079780460 & -0.4003004788 \\ \text { H164 } & 4.4067179747 & -0.9891556884 & -0.0444977211 \\ \text { C165 } & 4.4656403691 & 0.9997955765 & -2.8411297247 \\ \text { C166 } & 4.4849715649 & 0.9895904744 & -4.2481797070 \\ \text { H167 } & 3.5649857758 & 0.7767431022 & -4.7847517288 \\ \text { C168 } & 5.6709280059 & 1.2402526938 & -4.9317452725 \\ \text { H169 } & 5.6741011075 & 1.2262212582 & -6.0191425861 \\ \text { C170 } & 6.8490916988 & 1.5086640692 & -4.2326342127 \\ \text { H171 } & 7.7721834314 & 1.7052520677 & -4.7719189874 \\ \text { C172 } & 6.8335154522 & 1.5250623723 & -2.8365242301 \\ \text { H173 } & 7.7454604736 & 1.7386119635 & -2.2841042195 \\ \text { C174 } & 5.6550323808 & 1.2744380750 & -2.1399791165 \\ \text { H175 } & 5.6325031731 & 1.2960176711 & -1.0540967970\end{array}$

Table S3. Calculated coordinates for $C_{i}$-optimized 3.

$\begin{array}{llll}\text { Ta1 } & 1.2911648475 & -0.9060375040 & 1.5776917436 \\ \text { Ta2 } & 0.1946624062 & 1.9867967395 & 0.9777478551 \\ \text { Ta3 } & -1.8006018899 & -0.4403472166 & 1.2264390966 \\ \text { N4 } & -0.2011589879 & 0.3989993187 & 2.4775766130 \\ \text { N5 } & 2.1778538078 & 0.9198198000 & 0.8981969288 \\ \text { N6 } & 2.3002499997 & -1.6769515646 & 2.8395050872 \\ \text { N7 } & 0.4289794848 & 3.5628436745 & 1.7898415324 \\ \text { N8 } & 0.5332727277 & 2.1749552646 & -1.1753355401 \\ \text { N9 } & -1.8530160603 & 1.6610950601 & 0.4433484561 \\ \text { N10 } & -3.2770435890 & -0.7727683729 & 2.1804835401 \\ \text { O11 } & 0.0000000000 & 0.0000000000 & 0.0000000000 \\ \text { C12 } & -0.3424310167 & 0.7855539058 & 3.8465433840 \\ \text { C13 } & 0.7251525235 & 0.6850344440 & 4.7531456263 \\ \text { H14 } & 1.6774952876 & 0.2919746478 & 4.4132386797 \\ \text { C15 } & 0.5788387179 & 1.0871111795 & 6.0786034531 \\ \text { H16 } & 1.4287769112 & 0.9919975737 & 6.7519114027\end{array}$




\begin{tabular}{|c|c|c|c|}
\hline $\mathrm{C} 17$ & -0.6275812443 & 1.6130121014 & 6.5591595101 \\
\hline C19 & -1.6841085361 & 1.7235334522 & 5.6499248330 \\
\hline $\mathrm{H} 20$ & -2.6350340514 & 2.1366905029 & 5.9796717799 \\
\hline $\mathrm{C} 21$ & -1.5498306060 & 1.3223269160 & 4.3217914564 \\
\hline $\mathrm{H} 22$ & -2.3916169735 & 1.4330902904 & 3.6446786583 \\
\hline $\mathrm{C} 23$ & 3.3886462686 & 1.5664674697 & 1.3051399205 \\
\hline $\mathrm{C} 24$ & 4.3268774827 & 0.9421077476 & 2.1430934624 \\
\hline $\mathrm{H} 25$ & 4.1329924629 & -0.0599224912 & 2.5090142334 \\
\hline $\mathrm{C} 26$ & 5.4986886401 & 1.5979774183 & 2.5150054243 \\
\hline $\mathrm{H} 27$ & 6.2024931623 & 1.0829474631 & 3.1663462895 \\
\hline $\mathrm{C} 28$ & 5.7887414854 & 2.8957077482 & 2.0763129425 \\
\hline $\mathrm{C} 30$ & 4.8505731755 & 3.5138286056 & 1.2432723310 \\
\hline H31 & 5.0365167068 & 4.5224348311 & 0.8806015959 \\
\hline $\mathrm{C} 32$ & 3.6772660501 & 2.8677379041 & 0.8613451359 \\
\hline H33 & 2.9761878901 & 3.3808942896 & 0.2099760758 \\
\hline C34 & 3.0631748424 & -2.3096681518 & 3.8006435829 \\
\hline $\mathrm{C} 35$ & 4.3251305462 & -2.8469398738 & 3.4796256770 \\
\hline H36 & 4.6823034693 & -2.7764538159 & 2.4558381350 \\
\hline C37 & 5.0949324438 & -3.4651050016 & 4.4586755088 \\
\hline H38 & 6.0669180484 & -3.8734704296 & 4.1876985146 \\
\hline C39 & 4.6485127429 & -3.5748610291 & 5.7834319084 \\
\hline C41 & 3.3909165262 & -3.0450529941 & 6.0915039851 \\
\hline H42 & 3.0152246385 & -3.1198216538 & 7.1099338566 \\
\hline $\mathrm{C} 43$ & 2.6049133392 & -2.4237708887 & 5.1246461592 \\
\hline H44 & 1.6303959039 & -2.0201672554 & 5.3813229840 \\
\hline $\mathrm{C} 45$ & 0.6803204986 & 4.7463281449 & 2.4533142545 \\
\hline C46 & 0.7851879233 & 5.9606798097 & 1.7480731721 \\
\hline H47 & 0.6653354339 & 5.9538072128 & 0.6678601288 \\
\hline C48 & 1.0447687800 & 7.1463170957 & 2.4271574545 \\
\hline H49 & 1.1248537440 & 8.0732049429 & 1.8617478102 \\
\hline $\mathrm{C} 50$ & 1.2085279609 & 7.1728528364 & 3.8192369329 \\
\hline C52 & 1.1000130034 & 5.9611094505 & 4.5110397892 \\
\hline H53 & 1.2239026956 & 5.9517820150 & 5.5920631659 \\
\hline C54 & 0.8409315469 & 4.7636676538 & 3.8497377141 \\
\hline H55 & 0.7636834421 & 3.8304595620 & 4.4004203294 \\
\hline C56 & 0.6849611069 & 3.4468885786 & -1.8175585912 \\
\hline C57 & 1.8744999116 & 3.8281968863 & -2.4585646823 \\
\hline H58 & 2.7149549406 & 3.1432402812 & -2.4763790989 \\
\hline C59 & 1.9786946615 & 5.0671377700 & -3.0867943554 \\
\hline H60 & 2.9143781077 & 5.3271516470 & -3.5782416184 \\
\hline C61 & 0.9136881963 & 5.9763289754 & -3.1081210125 \\
\hline C63 & -0.2670831836 & 5.5943932632 & -2.4633341299 \\
\hline H64 & -1.1195797530 & 6.2702761865 & -2.4583662510 \\
\hline C65 & -0.3824716970 & 4.3600259482 & -1.8274836328 \\
\hline H66 & -1.3169087536 & 4.0996254539 & -1.3388287338 \\
\hline C67 & -2.9960720242 & 2.4961712174 & 0.6538720663 \\
\hline C68 & -2.8834638256 & 3.8181021374 & 1.1156470526 \\
\hline H69 & -1.9015159795 & 4.2283637661 & 1.3249526822 \\
\hline $\mathrm{C} 70$ & -4.0180044314 & 4.5979718975 & 1.3274792986 \\
\hline H71 & -3.8923770438 & 5.6163175990 & 1.6906191243 \\
\hline C72 & -5.3077168825 & 4.1041890963 & 1.0946677588 \\
\hline
\end{tabular}




\begin{tabular}{|c|c|c|c|}
\hline C74 & -5.4153249558 & 2.7896281328 & 0.6302729628 \\
\hline $\mathrm{H} 75$ & -6.3992085839 & 2.3654508190 & 0.4409053604 \\
\hline $\mathrm{C} 76$ & -4.2880096393 & 2.0014172238 & 0.4079279315 \\
\hline H77 & -4.4123257825 & 0.9843536128 & 0.0481292008 \\
\hline $\mathrm{C} 78$ & -4.4590461642 & -0.9963312059 & 2.8565684671 \\
\hline $\mathrm{C} 79$ & -4.5142339215 & -0.9385086131 & 4.2624224027 \\
\hline $\mathrm{H} 80$ & -3.6078986945 & -0.7083885318 & 4.8147810296 \\
\hline C81 & -5.7134569697 & -1.1654852465 & 4.9276512883 \\
\hline H82 & -5.7332314981 & -1.1145304369 & 6.0147919703 \\
\hline $\mathrm{C} 83$ & -6.8949467777 & -1.4581229395 & 4.2313949793 \\
\hline $\mathrm{C} 85$ & -6.8300570157 & -1.5173902556 & 2.8352998084 \\
\hline H86 & -7.7305330659 & -1.7453046585 & 2.2685139578 \\
\hline C87 & -5.6384260721 & -1.2928999391 & 2.1507471922 \\
\hline H88 & -5.6029597484 & -1.3516237355 & 1.0667500094 \\
\hline Ta89 & -1.2911648475 & 0.9060375040 & -1.5776917436 \\
\hline Ta90 & -0.1946624062 & -1.9867967395 & -0.9777478551 \\
\hline Ta91 & 1.8006018899 & 0.4403472166 & -1.2264390966 \\
\hline N92 & 0.2011589879 & -0.3989993187 & -2.4775766130 \\
\hline N93 & -2.1778538078 & -0.9198198000 & -0.8981969288 \\
\hline N94 & -2.3002499997 & 1.6769515646 & -2.8395050872 \\
\hline N95 & -0.4289794848 & -3.5628436745 & -1.7898415324 \\
\hline N96 & -0.5332727277 & -2.1749552646 & 1.1753355401 \\
\hline N97 & 1.8530160603 & -1.6610950601 & -0.4433484561 \\
\hline N98 & 3.2770435890 & 0.7727683729 & -2.1804835401 \\
\hline C99 & 0.3424310167 & -0.7855539058 & -3.8465433840 \\
\hline $\mathrm{C} 100$ & -0.7251525235 & -0.6850344440 & -4.7531456263 \\
\hline H101 & -1.6774952876 & -0.2919746478 & -4.4132386797 \\
\hline $\mathrm{C} 102$ & -0.5788387179 & -1.0871111795 & -6.0786034531 \\
\hline H103 & -1.4287769112 & -0.9919975737 & -6.7519114027 \\
\hline C104 & 0.6275812443 & -1.6130121014 & -6.5591595101 \\
\hline C106 & 1.6841085361 & -1.7235334522 & -5.6499248330 \\
\hline H107 & 2.6350340514 & -2.1366905029 & -5.9796717799 \\
\hline C108 & 1.5498306060 & -1.3223269160 & -4.3217914564 \\
\hline H109 & 2.3916169735 & -1.4330902904 & -3.6446786583 \\
\hline C110 & -3.3886462686 & -1.5664674697 & -1.3051399205 \\
\hline C111 & -4.3268774827 & -0.9421077476 & -2.1430934624 \\
\hline H112 & -4.1329924629 & 0.0599224912 & -2.5090142334 \\
\hline C113 & -5.4986886401 & -1.5979774183 & -2.5150054243 \\
\hline H114 & -6.2024931623 & -1.0829474631 & -3.1663462895 \\
\hline C115 & -5.7887414854 & -2.8957077482 & -2.0763129425 \\
\hline C117 & -4.8505731755 & -3.5138286056 & -1.2432723310 \\
\hline H118 & -5.0365167068 & -4.5224348311 & -0.8806015959 \\
\hline C119 & -3.6772660501 & -2.8677379041 & -0.8613451359 \\
\hline H120 & -2.9761878901 & -3.3808942896 & -0.2099760758 \\
\hline C121 & -3.0631748424 & 2.3096681518 & -3.8006435829 \\
\hline C122 & -4.3251305462 & 2.8469398738 & -3.4796256770 \\
\hline $\mathrm{H} 123$ & -4.6823034693 & 2.7764538159 & -2.4558381350 \\
\hline C124 & -5.0949324438 & 3.4651050016 & -4.4586755088 \\
\hline $\mathrm{H} 125$ & -6.0669180484 & 3.8734704296 & -4.1876985146 \\
\hline C126 & -4.6485127429 & 3.5748610291 & -5.7834319084 \\
\hline C128 & -3.3909165262 & 3.0450529941 & -6.0915039851 \\
\hline
\end{tabular}




\begin{tabular}{|c|c|c|c|}
\hline H129 & -3.0152246385 & 3.1198216538 & -7.1099338566 \\
\hline C130 & -2.6049133392 & 2.4237708887 & -5.1246461592 \\
\hline H131 & -1.6303959039 & 2.0201672554 & -5.3813229840 \\
\hline C132 & -0.6803204986 & -4.7463281449 & -2.4533142545 \\
\hline C133 & -0.7851879233 & -5.9606798097 & -1.7480731721 \\
\hline $\mathrm{H} 134$ & -0.6653354339 & -5.9538072128 & -0.6678601288 \\
\hline C135 & -1.0447687800 & -7.1463170957 & -2.4271574545 \\
\hline H136 & -1.1248537440 & -8.0732049429 & -1.8617478102 \\
\hline C137 & -1.2085279609 & -7.1728528364 & -3.8192369329 \\
\hline C139 & -1.1000130034 & -5.9611094505 & -4.5110397892 \\
\hline $\mathrm{H} 140$ & -1.2239026956 & -5.9517820150 & -5.5920631659 \\
\hline C141 & -0.8409315469 & -4.7636676538 & -3.8497377141 \\
\hline $\mathrm{H} 142$ & -0.7636834421 & -3.8304595620 & -4.4004203294 \\
\hline C143 & -0.6849611069 & -3.4468885786 & 1.8175585912 \\
\hline C144 & -1.8744999116 & -3.8281968863 & 2.4585646823 \\
\hline $\mathrm{H} 145$ & -2.7149549406 & -3.1432402812 & 2.4763790989 \\
\hline C146 & -1.9786946615 & -5.0671377700 & 3.0867943554 \\
\hline $\mathrm{H} 147$ & -2.9143781077 & -5.3271516470 & 3.5782416184 \\
\hline C148 & -0.9136881963 & -5.9763289754 & 3.1081210125 \\
\hline $\mathrm{C} 150$ & 0.2670831836 & -5.5943932632 & 2.4633341299 \\
\hline H151 & 1.1195797530 & -6.2702761865 & 2.4583662510 \\
\hline C152 & 0.3824716970 & -4.3600259482 & 1.8274836328 \\
\hline $\mathrm{H} 153$ & 1.3169087536 & -4.0996254539 & 1.3388287338 \\
\hline C154 & 2.9960720242 & -2.4961712174 & -0.6538720663 \\
\hline C155 & 2.8834638256 & -3.8181021374 & -1.1156470526 \\
\hline H156 & 1.9015159795 & -4.2283637661 & -1.3249526822 \\
\hline C157 & 4.0180044314 & -4.5979718975 & -1.3274792986 \\
\hline H158 & 3.8923770438 & -5.6163175990 & -1.6906191243 \\
\hline C159 & 5.3077168825 & -4.1041890963 & -1.0946677588 \\
\hline $\mathrm{C} 161$ & 5.4153249558 & -2.7896281328 & -0.6302729628 \\
\hline H162 & 6.3992085839 & -2.3654508190 & -0.4409053604 \\
\hline $\mathrm{C} 163$ & 4.2880096393 & -2.0014172238 & -0.4079279315 \\
\hline H164 & 4.4123257825 & -0.9843536128 & -0.0481292008 \\
\hline C165 & 4.4590461642 & 0.9963312059 & -2.8565684671 \\
\hline C166 & 4.5142339215 & 0.9385086131 & -4.2624224027 \\
\hline H167 & 3.6078986945 & 0.7083885318 & -4.8147810296 \\
\hline C168 & 5.7134569697 & 1.1654852465 & -4.9276512883 \\
\hline H169 & 5.7332314981 & 1.1145304369 & -6.0147919703 \\
\hline $\mathrm{C} 170$ & 6.8949467777 & 1.4581229395 & -4.2313949793 \\
\hline C172 & 6.8300570157 & 1.5173902556 & -2.8352998084 \\
\hline $\mathrm{H} 173$ & 7.7305330659 & 1.7453046585 & -2.2685139578 \\
\hline $\mathrm{C} 174$ & 5.6384260721 & 1.2928999391 & -2.1507471922 \\
\hline $\mathrm{H} 175$ & 5.6029597484 & 1.3516237355 & -1.0667500094 \\
\hline C176 & -5.5015980045 & 4.2453292131 & -6.8357063568 \\
\hline H177 & -5.0045661941 & 4.2474486727 & -7.8112742511 \\
\hline $\mathrm{H} 178$ & -6.4666545816 & 3.7362231811 & -6.9555025118 \\
\hline H179 & -5.7207763898 & 5.2879753600 & -6.5719373836 \\
\hline C179 & 5.5015980045 & -4.2453292131 & 6.8357063568 \\
\hline H180 & 5.0045661941 & -4.2474486727 & 7.8112742511 \\
\hline H181 & 5.7207763898 & -5.2879753600 & 6.5719373836 \\
\hline H182 & 6.4666545816 & -3.7362231811 & 6.9555025118 \\
\hline
\end{tabular}




\begin{tabular}{|c|c|c|c|}
\hline C182 & 1.4959294264 & 8.4696285393 & 4.5409598215 \\
\hline H183 & 1.5970775267 & 8.3123358769 & 5.6198007434 \\
\hline H184 & 2.4257038817 & 8.9311842890 & 4.1839530500 \\
\hline H185 & 0.6947402753 & 9.2037488369 & 4.3857320531 \\
\hline C185 & -1.4959294264 & -8.4696285393 & -4.5409598215 \\
\hline H186 & -1.5970775267 & -8.3123358769 & -5.6198007434 \\
\hline H187 & -0.6947402753 & -9.2037488369 & -4.3857320531 \\
\hline H188 & -2.4257038817 & -8.9311842890 & -4.1839530500 \\
\hline C188 & 8.1899046111 & 1.6999672803 & -4.9724162812 \\
\hline H189 & 9.0108258552 & 1.9195816647 & -4.2820859703 \\
\hline H190 & 8.4815741546 & 0.8251809860 & -5.5678680478 \\
\hline H191 & 8.1044327181 & 2.5451648523 & -5.6674411227 \\
\hline C191 & -8.1899046111 & -1.6999672803 & 4.9724162812 \\
\hline H192 & -9.0108258552 & -1.9195816647 & 4.2820859703 \\
\hline H193 & -8.1044327181 & -2.5451648523 & 5.6674411227 \\
\hline H194 & -8.4815741546 & -0.8251809860 & 5.5678680478 \\
\hline C194 & -0.7713025129 & 2.0475853472 & 7.9996106520 \\
\hline $\mathrm{H} 195$ & -1.7727699537 & 2.4423636307 & 8.1991838199 \\
\hline H196 & -0.5990796501 & 1.2127456598 & 8.6910300504 \\
\hline H197 & -0.0473606009 & 2.8309905165 & 8.2577835273 \\
\hline C197 & 0.7713025129 & -2.0475853472 & -7.9996106520 \\
\hline H198 & 1.7727699537 & -2.4423636307 & -8.1991838199 \\
\hline H199 & 0.0473606009 & -2.8309905165 & -8.2577835273 \\
\hline $\mathrm{H} 200$ & 0.5990796501 & -1.2127456598 & -8.6910300504 \\
\hline C200 & -6.5285956453 & 4.9604191240 & 1.3401703038 \\
\hline H201 & -7.4503585970 & 4.4152369478 & 1.1124187752 \\
\hline H202 & -6.5856382713 & 5.2874731518 & 2.3861349206 \\
\hline $\mathrm{H} 203$ & -6.5159235589 & 5.8668083283 & 0.7214065633 \\
\hline C203 & 6.5285956453 & -4.9604191240 & -1.3401703038 \\
\hline H204 & 7.4503585970 & -4.4152369478 & -1.1124187752 \\
\hline H205 & 6.5159235589 & -5.8668083283 & -0.7214065633 \\
\hline H206 & 6.5856382713 & -5.2874731518 & -2.3861349206 \\
\hline C206 & 7.0631985444 & 3.5959764205 & 2.4882629828 \\
\hline H207 & 7.1295861584 & 4.5944841329 & 2.0440892703 \\
\hline H208 & 7.1237929614 & 3.7125709534 & 3.5777655581 \\
\hline H209 & 7.9520741669 & 3.0327914833 & 2.1762325576 \\
\hline C209 & -1.0379754058 & -7.3108888447 & 3.8061984799 \\
\hline $\mathrm{H} 210$ & -0.1156260964 & -7.8941201379 & 3.7181354771 \\
\hline $\mathrm{H} 211$ & -1.2504362603 & -7.1858040795 & 4.8756001541 \\
\hline H212 & -1.8543698952 & -7.9118204756 & 3.3856529233 \\
\hline C212 & -7.0631985444 & -3.5959764205 & -2.4882629828 \\
\hline $\mathrm{H} 213$ & -7.1295861584 & -4.5944841329 & -2.0440892703 \\
\hline $\mathrm{H} 214$ & -7.9520741669 & -3.0327914833 & -2.1762325576 \\
\hline $\mathrm{H} 215$ & -7.1237929614 & -3.7125709534 & -3.5777655581 \\
\hline $\mathrm{C} 215$ & 1.0379754058 & 7.3108888447 & -3.8061984799 \\
\hline H216 & 0.1156260964 & 7.8941201379 & -3.7181354771 \\
\hline $\mathrm{H} 217$ & 1.8543698952 & 7.9118204756 & -3.3856529233 \\
\hline H218 & 1.2504362603 & 7.1858040795 & -4.8756001541 \\
\hline
\end{tabular}


Table S4. Calculated coordinates for $C_{i}$-optimized 4.

\begin{tabular}{|c|c|c|c|}
\hline Ta1 & -0.0310201169 & -2.2135575039 & -0.2663058695 \\
\hline $\mathrm{Ta} 2$ & 0.9670547207 & 0.2294047805 & -1.9873534119 \\
\hline Ta3 & 1.9949174152 & -0.1471941447 & 0.9666879508 \\
\hline N4 & 1.9185981053 & -1.4027886316 & -0.8248383617 \\
\hline N5 & -0.7066669223 & -1.2703159575 & -2.0656468044 \\
\hline N6 & -0.0690089018 & -3.9994856974 & -0.4142555644 \\
\hline N7 & 1.6899554931 & 0.3188865310 & -3.6227105600 \\
\hline N8 & -0.6878318399 & 1.6416965318 & -1.7927498521 \\
\hline N9 & 1.9633520882 & 1.4857006301 & -0.5639314384 \\
\hline N10 & 3.6085774166 & -0.2210067354 & 1.7425857746 \\
\hline $\mathrm{O} 11$ & 0.0000000000 & 0.0000000000 & 0.0000000000 \\
\hline $\mathrm{C} 12$ & 3.0652157415 & -2.0376422353 & -1.3975894774 \\
\hline $\mathrm{C} 13$ & 2.9663823852 & -3.2755263779 & -2.0576381299 \\
\hline H14 & 2.0019546233 & -3.7691190704 & -2.1176360329 \\
\hline $\mathrm{C} 15$ & 4.0783226181 & -3.8738221160 & -2.6377776170 \\
\hline H16 & 3.9935120642 & -4.8308856820 & -3.1441614856 \\
\hline $\mathrm{C} 17$ & 5.3310039985 & -3.2491209091 & -2.5868536006 \\
\hline C19 & 5.4474216802 & -2.0129529215 & -1.9454338233 \\
\hline $\mathrm{H} 20$ & 6.3974131545 & -1.4928073596 & -1.8941234254 \\
\hline $\mathrm{C} 21$ & 4.3248295006 & -1.4222362944 & -1.3629367151 \\
\hline $\mathrm{H} 22$ & 4.4371310306 & -0.4564309451 & -0.8799274226 \\
\hline $\mathrm{C} 23$ & -1.1527839344 & -1.8219545575 & -3.3070730385 \\
\hline $\mathrm{C} 24$ & -1.3888915105 & -3.1972474051 & -3.4830803929 \\
\hline $\mathrm{H} 25$ & -1.2098643322 & -3.8798933579 & -2.6589439670 \\
\hline $\mathrm{C} 26$ & -1.8453528328 & -3.6956942748 & -4.6985304174 \\
\hline $\mathrm{H} 27$ & -2.0264353074 & -4.7585824790 & -4.8291267969 \\
\hline $\mathrm{C} 28$ & -2.0768924847 & -2.8386978554 & -5.7811928733 \\
\hline $\mathrm{C} 30$ & -1.8403520267 & -1.4696198979 & -5.6274402823 \\
\hline H31 & -2.0031463146 & -0.7741806152 & -6.4430914272 \\
\hline $\mathrm{C} 32$ & -1.3868733135 & -0.9785972977 & -4.4043456312 \\
\hline H33 & -1.2157105344 & 0.0888456170 & -4.3046177030 \\
\hline C34 & -0.0925319726 & -5.3803404174 & -0.4586471368 \\
\hline $\mathrm{C} 35$ & -1.3137741183 & -6.0860871096 & -0.4856273041 \\
\hline H36 & -2.2456968854 & -5.5283170321 & -0.4538065978 \\
\hline C37 & -1.3279496862 & -7.4718571005 & -0.5397114962 \\
\hline H38 & -2.2642514165 & -8.0216894752 & -0.5587161165 \\
\hline C39 & -0.1282612159 & -8.1960541987 & -0.5695657998 \\
\hline $\mathrm{C} 40$ & 1.0906260589 & -7.5111145191 & -0.5373765006 \\
\hline H41 & 2.0338582862 & -8.0456147012 & -0.5540535087 \\
\hline $\mathrm{C} 42$ & 1.1021753228 & -6.1183550650 & -0.4803442151 \\
\hline H43 & 2.0479080080 & -5.5863949959 & -0.4498896829 \\
\hline C44 & 2.1939407148 & 0.2340820742 & -4.9041651202 \\
\hline $\mathrm{C} 45$ & 1.9336751207 & 1.2329558022 & -5.8646562135 \\
\hline H46 & 1.3304259202 & 2.0917411496 & -5.5825032642 \\
\hline $\mathrm{C} 47$ & 2.4250159456 & 1.1145163159 & -7.1569352520 \\
\hline H48 & 2.2246508651 & 1.8764687577 & -7.9044024196 \\
\hline C49 & 3.1857433730 & -0.0022118766 & -7.5293791412 \\
\hline C51 & 3.4597923581 & -0.9969144480 & -6.5849782433 \\
\hline H52 & 4.0454279693 & -1.8716372708 & -6.8454439408 \\
\hline
\end{tabular}




\begin{tabular}{|c|c|c|c|}
\hline C53 & 2.9671397581 & -0.8744278643 & -5.2878303745 \\
\hline H54 & 3.1661854478 & -1.6523814924 & -4.5564832938 \\
\hline C55 & -0.9304083343 & 2.6638163728 & -2.7652349283 \\
\hline C56 & -2.1222969601 & 2.7351672851 & -3.5082116044 \\
\hline H57 & -2.8957495376 & 1.9910783264 & -3.3499367613 \\
\hline C58 & -2.3331507894 & 3.7579327956 & -4.4254293449 \\
\hline H59 & -3.2586876712 & 3.8135897554 & -4.9908548760 \\
\hline C60 & -1.3630998834 & 4.7462378683 & -4.6312852902 \\
\hline C62 & -0.1677506729 & 4.6867961313 & -3.9086177280 \\
\hline H63 & 0.6075684415 & 5.4335210588 & -4.0380673530 \\
\hline C64 & 0.0369936661 & 3.6541530788 & -2.9942467825 \\
\hline H65 & 0.9711297497 & 3.6284033399 & -2.4411898493 \\
\hline C66 & 3.0964961566 & 2.3411112805 & -0.7220735905 \\
\hline C67 & 3.6515489269 & 2.6218369203 & -1.9842779016 \\
\hline H68 & 3.2106150183 & 2.1785093696 & -2.8713470771 \\
\hline C69 & 4.7715331518 & 3.4341368323 & -2.1112068460 \\
\hline $\mathrm{H} 70$ & 5.1991214520 & 3.6409418914 & -3.0878976494 \\
\hline C71 & 5.3803289597 & 3.9941920822 & -0.9818056794 \\
\hline C73 & 4.8390543708 & 3.7344973594 & 0.2808753589 \\
\hline H74 & 5.2838174348 & 4.1462062999 & 1.1800387322 \\
\hline $\mathrm{C} 75$ & 3.7099294810 & 2.9232238474 & 0.3978131600 \\
\hline H76 & 3.3071513267 & 2.7314176963 & 1.3875990420 \\
\hline $\mathrm{C} 77$ & 4.8568155589 & -0.1774425727 & 2.3302298425 \\
\hline $\mathrm{C} 78$ & 5.8806253717 & -1.0698954393 & 1.9488755388 \\
\hline H79 & 5.6769225749 & -1.8051572661 & 1.1762056649 \\
\hline C80 & 7.1334393615 & -1.0021800512 & 2.5390606575 \\
\hline H81 & 7.9271290569 & -1.6841872659 & 2.2486388268 \\
\hline C82 & 7.4042470958 & -0.0453340210 & 3.5269781637 \\
\hline C84 & 6.3971699098 & 0.8400814187 & 3.9234935121 \\
\hline H85 & 6.5775391954 & 1.5860837664 & 4.6896770590 \\
\hline C86 & 5.1383962081 & 0.7699371548 & 3.3284898346 \\
\hline H87 & 4.3545395745 & 1.4549003403 & 3.6377165308 \\
\hline Ta88 & 0.0310201169 & 2.2135575039 & 0.2663058695 \\
\hline Ta89 & -0.9670547207 & -0.2294047805 & 1.9873534119 \\
\hline Ta90 & -1.9949174152 & 0.1471941447 & -0.9666879508 \\
\hline N91 & -1.9185981053 & 1.4027886316 & 0.8248383617 \\
\hline N92 & 0.7066669223 & 1.2703159575 & 2.0656468044 \\
\hline N93 & 0.0690089018 & 3.9994856974 & 0.4142555644 \\
\hline N94 & -1.6899554931 & -0.3188865310 & 3.6227105600 \\
\hline N95 & 0.6878318399 & -1.6416965318 & 1.7927498521 \\
\hline N96 & -1.9633520882 & -1.4857006301 & 0.5639314384 \\
\hline N97 & -3.6085774166 & 0.2210067354 & -1.7425857746 \\
\hline C98 & -3.0652157415 & 2.0376422353 & 1.3975894774 \\
\hline C99 & -2.9663823852 & 3.2755263779 & 2.0576381299 \\
\hline H100 & -2.0019546233 & 3.7691190704 & 2.1176360329 \\
\hline C101 & -4.0783226181 & 3.8738221160 & 2.6377776170 \\
\hline H102 & -3.9935120642 & 4.8308856820 & 3.1441614856 \\
\hline $\mathrm{C} 103$ & -5.3310039985 & 3.2491209091 & 2.5868536006 \\
\hline C105 & -5.4474216802 & 2.0129529215 & 1.9454338233 \\
\hline H106 & -6.3974131545 & 1.4928073596 & 1.8941234254 \\
\hline C107 & -4.3248295006 & 1.4222362944 & 1.3629367151 \\
\hline
\end{tabular}




\begin{tabular}{|c|c|c|c|}
\hline H108 & -4.4371310306 & 0.4564309451 & 0.8799274226 \\
\hline C109 & 1.1527839344 & 1.8219545575 & 3.3070730385 \\
\hline C110 & 1.3888915105 & 3.1972474051 & 3.4830803929 \\
\hline H111 & 1.2098643322 & 3.8798933579 & 2.6589439670 \\
\hline C112 & 1.8453528328 & 3.6956942748 & 4.6985304174 \\
\hline $\mathrm{H} 113$ & 2.0264353074 & 4.7585824790 & 4.8291267969 \\
\hline C114 & 2.0768924847 & 2.8386978554 & 5.7811928733 \\
\hline C116 & 1.8403520267 & 1.4696198979 & 5.6274402823 \\
\hline H117 & 2.0031463146 & 0.7741806152 & 6.4430914272 \\
\hline C118 & 1.3868733135 & 0.9785972977 & 4.4043456312 \\
\hline H119 & 1.2157105344 & -0.0888456170 & 4.3046177030 \\
\hline C120 & 0.0925319726 & 5.3803404174 & 0.4586471368 \\
\hline C121 & 1.3137741183 & 6.0860871096 & 0.4856273041 \\
\hline $\mathrm{H} 122$ & 2.2456968854 & 5.5283170321 & 0.4538065978 \\
\hline C123 & 1.3279496862 & 7.4718571005 & 0.5397114962 \\
\hline $\mathrm{H} 124$ & 2.2642514165 & 8.0216894752 & 0.5587161165 \\
\hline C125 & 0.1282612159 & 8.1960541987 & 0.5695657998 \\
\hline C126 & -1.0906260589 & 7.5111145191 & 0.5373765006 \\
\hline $\mathrm{H} 127$ & -2.0338582862 & 8.0456147012 & 0.5540535087 \\
\hline C128 & -1.1021753228 & 6.1183550650 & 0.4803442151 \\
\hline H129 & -2.0479080080 & 5.5863949959 & 0.4498896829 \\
\hline C130 & -2.1939407148 & -0.2340820742 & 4.9041651202 \\
\hline C131 & -1.9336751207 & -1.2329558022 & 5.8646562135 \\
\hline H132 & -1.3304259202 & -2.0917411496 & 5.5825032642 \\
\hline C133 & -2.4250159456 & -1.1145163159 & 7.1569352520 \\
\hline $\mathrm{H} 134$ & -2.2246508651 & -1.8764687577 & 7.9044024196 \\
\hline C135 & -3.1857433730 & 0.0022118766 & 7.5293791412 \\
\hline C137 & -3.4597923581 & 0.9969144480 & 6.5849782433 \\
\hline H138 & -4.0454279693 & 1.8716372708 & 6.8454439408 \\
\hline C139 & -2.9671397581 & 0.8744278643 & 5.2878303745 \\
\hline H140 & -3.1661854478 & 1.6523814924 & 4.5564832938 \\
\hline C141 & 0.9304083343 & -2.6638163728 & 2.7652349283 \\
\hline C142 & 2.1222969601 & -2.7351672851 & 3.5082116044 \\
\hline $\mathrm{H} 143$ & 2.8957495376 & -1.9910783264 & 3.3499367613 \\
\hline C144 & 2.3331507894 & -3.7579327956 & 4.4254293449 \\
\hline $\mathrm{H} 145$ & 3.2586876712 & -3.8135897554 & 4.9908548760 \\
\hline C146 & 1.3630998834 & -4.7462378683 & 4.6312852902 \\
\hline C148 & 0.1677506729 & -4.6867961313 & 3.9086177280 \\
\hline H149 & -0.6075684415 & -5.4335210588 & 4.0380673530 \\
\hline C150 & -0.0369936661 & -3.6541530788 & 2.9942467825 \\
\hline H151 & -0.9711297497 & -3.6284033399 & 2.4411898493 \\
\hline C152 & -3.0964961566 & -2.3411112805 & 0.7220735905 \\
\hline C153 & -3.6515489269 & -2.6218369203 & 1.9842779016 \\
\hline H154 & -3.2106150183 & -2.1785093696 & 2.8713470771 \\
\hline C155 & -4.7715331518 & -3.4341368323 & 2.1112068460 \\
\hline H156 & -5.1991214520 & -3.6409418914 & 3.0878976494 \\
\hline $\mathrm{C} 157$ & -5.3803289597 & -3.9941920822 & 0.9818056794 \\
\hline C159 & -4.8390543708 & -3.7344973594 & -0.2808753589 \\
\hline $\mathrm{H} 160$ & -5.2838174348 & -4.1462062999 & -1.1800387322 \\
\hline $\mathrm{C} 161$ & -3.7099294810 & -2.9232238474 & -0.3978131600 \\
\hline H162 & -3.3071513267 & -2.7314176963 & -1.3875990420 \\
\hline
\end{tabular}




\begin{tabular}{|c|c|c|c|}
\hline $\mathrm{C} 163$ & -4.8568155589 & 0.1774425727 & -2.3302298425 \\
\hline $\mathrm{C} 164$ & -5.8806253717 & 1.0698954393 & -1.9488755388 \\
\hline H165 & -5.6769225749 & 1.8051572661 & -1.1762056649 \\
\hline $\mathrm{C} 166$ & -7.1334393615 & 1.0021800512 & -2.5390606575 \\
\hline H167 & -7.9271290569 & 1.6841872659 & -2.2486388268 \\
\hline C168 & -7.4042470958 & 0.0453340210 & -3.5269781637 \\
\hline $\mathrm{C} 170$ & -6.3971699098 & -0.8400814187 & -3.9234935121 \\
\hline H171 & -6.5775391954 & -1.5860837664 & -4.6896770590 \\
\hline C172 & -5.1383962081 & -0.7699371548 & -3.3284898346 \\
\hline $\mathrm{H} 173$ & -4.3545395745 & -1.4549003403 & -3.6377165308 \\
\hline $\mathrm{O} 175$ & 0.2584517277 & 9.5551139724 & 0.6269031380 \\
\hline $\mathrm{O} 176$ & -0.2584517277 & -9.5551139724 & -0.6269031380 \\
\hline $\mathrm{C} 177$ & -0.9206702479 & 10.3373855206 & 0.6675557193 \\
\hline $\mathrm{H} 178$ & -0.5909840292 & 11.3774219831 & 0.7124993446 \\
\hline H179 & -1.5360859034 & 10.1915875459 & -0.2306352662 \\
\hline H180 & -1.5268867064 & 10.1112724633 & 1.5552731570 \\
\hline C181 & 0.9206702479 & -10.3373855206 & -0.6675557193 \\
\hline H182 & 0.5909840292 & -11.3774219831 & -0.7124993446 \\
\hline $\mathrm{H} 183$ & 1.5268867064 & -10.1112724633 & -1.5552731570 \\
\hline H184 & 1.5360859034 & -10.1915875459 & 0.2306352662 \\
\hline O186 & 3.6056493882 & -0.0274028284 & -8.8304342520 \\
\hline $\mathrm{O} 187$ & -3.6056493882 & 0.0274028284 & 8.8304342520 \\
\hline O188 & 8.6737188417 & -0.0618995516 & 4.0337351866 \\
\hline O189 & -8.6737188417 & 0.0618995516 & -4.0337351866 \\
\hline O190 & 6.4827161258 & 4.7655623602 & -1.2171288806 \\
\hline O191 & 2.5318481469 & 3.4269548795 & 6.9274112472 \\
\hline O192 & -6.3578767948 & 3.9173705031 & 3.1918777893 \\
\hline $\mathrm{O} 193$ & -1.6751329272 & 5.7121917346 & -5.5451655131 \\
\hline O194 & 1.6751329272 & -5.7121917346 & 5.5451655131 \\
\hline $\mathrm{O} 195$ & -2.5318481469 & -3.4269548795 & -6.9274112472 \\
\hline O196 & -6.4827161258 & -4.7655623602 & 1.2171288806 \\
\hline O197 & 6.3578767948 & -3.9173705031 & -3.1918777893 \\
\hline C198 & 9.0095663792 & 0.8890334827 & 5.0261248140 \\
\hline H199 & 10.0549012337 & 0.7025057275 & 5.2812532131 \\
\hline H200 & 8.9059434377 & 1.9175859272 & 4.6538666754 \\
\hline H201 & 8.3916250761 & 0.7725269695 & 5.9268367979 \\
\hline C201 & -9.0095663792 & -0.8890334827 & -5.0261248140 \\
\hline H202 & -10.0549012337 & -0.7025057275 & -5.2812532131 \\
\hline H203 & -8.3916250761 & -0.7725269695 & -5.9268367979 \\
\hline H204 & -8.9059434377 & -1.9175859272 & -4.6538666754 \\
\hline C204 & -7.6389432867 & 3.3144798071 & 3.1854851332 \\
\hline H205 & -8.2995259288 & 4.0046573842 & 3.7145533086 \\
\hline H206 & -8.0136254937 & 3.1673792256 & 2.1634602496 \\
\hline H207 & -7.6338815022 & 2.3468989308 & 3.7056983515 \\
\hline C207 & 2.7879965910 & 2.5949753825 & 8.0442472370 \\
\hline $\mathrm{H} 208$ & 3.1371015400 & 3.2569565636 & 8.8395969908 \\
\hline H209 & 1.8813947380 & 2.0734579282 & 8.3793068036 \\
\hline $\mathrm{H} 210$ & 3.5659772193 & 1.8504512811 & 7.8251072310 \\
\hline $\mathrm{C} 210$ & 7.1453297022 & 5.3379593197 & -0.1051763550 \\
\hline H211 & 7.9867671579 & 5.9018064177 & -0.5134291485 \\
\hline H212 & 6.4890277889 & 6.0209551304 & 0.4514246422 \\
\hline
\end{tabular}




$\begin{array}{llll}\text { H213 } & 7.5236488836 & 4.5682336830 & 0.5812711651 \\ \text { C213 } & -0.7301818674 & 6.7414168337 & -5.7741278107 \\ \text { H214 } & -1.1753663485 & 7.3976642117 & -6.5249184018 \\ \text { H215 } & -0.5297169544 & 7.3183191421 & -4.8611314016 \\ \text { H216 } & 0.2184966733 & 6.3432560717 & -6.1594080436 \\ \text { C216 } & -4.3517264012 & 1.1474604757 & 9.2682852164 \\ \text { H217 } & -4.5752196043 & 0.9680589638 & 10.3221408289 \\ \text { H218 } & -3.7774427460 & 2.0793631894 & 9.1733837498 \\ \text { H219 } & -5.2934383706 & 1.2522735528 & 8.7125304848 \\ \text { C219 } & 4.3517264012 & -1.1474604757 & -9.2682852164 \\ \text { H220 } & 4.5752196043 & -0.9680589638 & -10.3221408289 \\ \text { H221 } & 5.2934383706 & -1.2522735528 & -8.7125304848 \\ \text { H222 } & 3.7774427460 & -2.0793631894 & -9.1733837498 \\ \text { C222 } & -2.7879965910 & -2.5949753825 & -8.0442472370 \\ \text { H223 } & -3.1371015400 & -3.2569565636 & -8.8395969908 \\ \text { H224 } & -3.5659772193 & -1.8504512811 & -7.8251072310 \\ \text { H225 } & -1.8813947380 & -2.0734579282 & -8.3793068036 \\ \text { C225 } & 0.7301818674 & -6.7414168337 & 5.7741278107 \\ \text { H226 } & 1.1753663485 & -7.3976642117 & 6.5249184018 \\ \text { H227 } & -0.2184966733 & -6.3432560717 & 6.1594080436 \\ \text { H228 } & 0.5297169544 & -7.3183191421 & 4.8611314016 \\ \text { C228 } & -7.1453297022 & -5.3379593197 & 0.1051763550 \\ \text { H229 } & -7.9867671579 & -5.9018064177 & 0.5134291485 \\ \text { H230 } & -7.5236488836 & -4.5682336830 & -0.5812711651 \\ \text { H231 } & -6.4890277889 & -6.0209551304 & -0.4514246422 \\ \text { C231 } & 7.6389432867 & -3.3144798071 & -3.1854851332 \\ \text { H232 } & 8.2995259288 & -4.0046573842 & -3.7145533086 \\ \text { H233 } & 7.6338815022 & -2.3468989308 & -3.7056983515 \\ \text { H234 } & 8.0136254937 & -3.1673792256 & -2.1634602496\end{array}$

Table S5. Calculated coordinates for $C_{i}$-optimized 9.

$\begin{array}{llll}\text { Ta1 } & 1.2882211810 & -0.9075001947 & 1.5763630727 \\ \text { Ta2 } & 0.1984084797 & 1.9882340609 & 0.9814400156 \\ \text { Ta3 } & -1.8073976992 & -0.4306944224 & 1.2281612329 \\ \text { N4 } & -0.1939555675 & 0.4078395027 & 2.4824362570 \\ \text { N5 } & 2.1654450741 & 0.9554526937 & 0.8829072430 \\ \text { N6 } & 2.2914322480 & -1.6918403091 & 2.8305734755 \\ \text { N7 } & 0.4219645645 & 3.5664660176 & 1.7868233748 \\ \text { N8 } & 0.4903632910 & 2.1739408468 & -1.1838290102 \\ \text { N9 } & -1.8777272505 & 1.6308505219 & 0.4445395926 \\ \text { N10 } & -3.2928120949 & -0.7443951709 & 2.1711652948 \\ \text { O11 } & 0.0000000000 & 0.0000000000 & 0.0000000000 \\ \text { C12 } & -0.3015870995 & 0.7854559896 & 3.8546849818 \\ \text { C13 } & 0.7854378329 & 0.7060555517 & 4.7361004917 \\ \text { H14 } & 1.7476588765 & 0.3361185875 & 4.3999906969 \\ \text { C15 } & 0.6177129084 & 1.1124503698 & 6.0590523965 \\ \text { H16 } & 1.4570912321 & 1.0499508453 & 6.7496950498 \\ \text { N17 } & -0.5257187806 & 1.5898132451 & 6.5635420370 \\ \text { C18 } & -1.5576154842 & 1.6709007668 & 5.7151119222 \\ \text { H19 } & -2.4859188956 & 2.0655566822 & 6.1247869876\end{array}$




\begin{tabular}{|c|c|c|c|}
\hline $\mathrm{C} 20$ & -1.5030583206 & 1.2909804832 & 4.3757034217 \\
\hline $\mathrm{H} 21$ & -2.3822965176 & 1.3918086412 & 3.7473981059 \\
\hline $\mathrm{C} 22$ & 3.3784557938 & 1.5868353923 & 1.2980240258 \\
\hline $\mathrm{C} 23$ & 4.2957566268 & 0.9612348343 & 2.1534589413 \\
\hline $\mathrm{H} 24$ & 4.1013202165 & -0.0286133245 & 2.5490831499 \\
\hline $\mathrm{C} 25$ & 5.4689563045 & 1.6326627165 & 2.4969657226 \\
\hline $\mathrm{H} 26$ & 6.1871768388 & 1.1519188683 & 3.1594080522 \\
\hline $\mathrm{N} 27$ & 5.7947682408 & 2.8548911463 & 2.0649802577 \\
\hline $\mathrm{C} 28$ & 4.9102281170 & 3.4512851780 & 1.2559660030 \\
\hline $\mathrm{H} 29$ & 5.1734835885 & 4.4493216137 & 0.9103468465 \\
\hline $\mathrm{C} 30$ & 3.7107059294 & 2.8745518361 & 0.8461208088 \\
\hline H31 & 3.0468479967 & 3.4215786927 & 0.1839814035 \\
\hline $\mathrm{C} 32$ & 3.0392159625 & -2.3534409133 & 3.7828318416 \\
\hline C33 & 4.3169132837 & -2.8537744465 & 3.4809867556 \\
\hline H34 & 4.7300950402 & -2.7377916797 & 2.4839937170 \\
\hline $\mathrm{C} 35$ & 5.0274250250 & -3.5105116407 & 4.4823510033 \\
\hline H36 & 6.0183768499 & -3.9079890915 & 4.2693210135 \\
\hline N37 & 4.5734384820 & -3.7001588734 & 5.7269846464 \\
\hline $\mathrm{C} 38$ & 3.3527624358 & -3.2234162410 & 6.0015912374 \\
\hline H39 & 2.9925698976 & -3.3887849622 & 7.0155599466 \\
\hline $\mathrm{C} 40$ & 2.5493671003 & -2.5514230680 & 5.0846093593 \\
\hline H41 & 1.5659153621 & -2.1863443472 & 5.3619036608 \\
\hline $\mathrm{C} 42$ & 0.6439260462 & 4.7724714963 & 2.4196200238 \\
\hline $\mathrm{C} 43$ & 0.7250716728 & 5.9690531404 & 1.6883177850 \\
\hline H44 & 0.6159094194 & 5.9612369821 & 0.6082717555 \\
\hline $\mathrm{C} 45$ & 0.9501728906 & 7.1556096522 & 2.3813886034 \\
\hline H46 & 1.0190026118 & 8.0950897981 & 1.8354171055 \\
\hline N47 & 1.0939371508 & 7.2360410767 & 3.7095728406 \\
\hline $\mathrm{C} 48$ & 1.0137625676 & 6.0883862129 & 4.3951326705 \\
\hline H49 & 1.1328218994 & 6.1673642693 & 5.4743648541 \\
\hline $\mathrm{C} 50$ & 0.7931249231 & 4.8427181479 & 3.8146516492 \\
\hline H51 & 0.7370514736 & 3.9431305991 & 4.4191999024 \\
\hline C52 & 0.6567981332 & 3.4405083560 & -1.8258116035 \\
\hline C53 & 1.8456186689 & 3.8081784781 & -2.4702076842 \\
\hline H54 & 2.6867451846 & 3.1264700046 & -2.5158260793 \\
\hline C55 & 1.9312999658 & 5.0651854868 & -3.0687174768 \\
\hline H56 & 2.8523595212 & 5.3572714956 & -3.5705969890 \\
\hline N57 & 0.9449668698 & 5.9674114794 & -3.0777164955 \\
\hline C58 & -0.1889005110 & 5.6093830292 & -2.4626984148 \\
\hline H59 & -0.9935517130 & 6.3426071384 & -2.4750891003 \\
\hline C60 & -0.3832175306 & 4.3844796306 & -1.8291704693 \\
\hline H61 & -1.3330034006 & 4.1691601920 & -1.3493584265 \\
\hline C62 & -3.0155165956 & 2.4711414699 & 0.6449911988 \\
\hline C63 & -2.9027931726 & 3.7892719950 & 1.1078208315 \\
\hline H64 & -1.9335654254 & 4.2156940227 & 1.3392815471 \\
\hline C65 & -4.0599344048 & 4.5485795678 & 1.2826281878 \\
\hline H66 & -3.9784085233 & 5.5726340271 & 1.6432446735 \\
\hline N67 & -5.2983086906 & 4.1077940678 & 1.0395024853 \\
\hline C68 & -5.4001063888 & 2.8481028395 & 0.5986472161 \\
\hline H69 & -6.4093827158 & 2.4874166903 & 0.4068090172 \\
\hline C70 & -4.3143092234 & 2.0033127711 & 0.3821987165 \\
\hline
\end{tabular}




\begin{tabular}{|c|c|c|c|}
\hline H71 & -4.4791632858 & 0.9934140521 & 0.0204741125 \\
\hline $\mathrm{C} 72$ & -4.4690204915 & -0.9683479347 & 2.8567054920 \\
\hline C73 & -4.5030799149 & -0.9717767014 & 4.2610826112 \\
\hline H74 & -3.5976859943 & -0.7923042403 & 4.8317023260 \\
\hline $\mathrm{C} 75$ & -5.7203268288 & -1.2042167297 & 4.8958177009 \\
\hline H76 & -5.7698797448 & -1.2091679813 & 5.9834385808 \\
\hline N77 & -6.8731950237 & -1.4305146523 & 4.2545166764 \\
\hline $\mathrm{C} 78$ & -6.8278336067 & -1.4298208632 & 2.9164975884 \\
\hline H79 & -7.7708222058 & -1.6189066470 & 2.4063252191 \\
\hline $\mathrm{C} 80$ & -5.6725202591 & -1.2085440991 & 2.1720324171 \\
\hline H81 & -5.6936168109 & -1.2296742195 & 1.0872442333 \\
\hline Ta82 & -1.2882211810 & 0.9075001947 & -1.5763630727 \\
\hline Ta83 & -0.1984084797 & -1.9882340609 & -0.9814400156 \\
\hline Ta84 & 1.8073976992 & 0.4306944224 & -1.2281612329 \\
\hline N85 & 0.1939555675 & -0.4078395027 & -2.4824362570 \\
\hline N86 & -2.1654450741 & -0.9554526937 & -0.8829072430 \\
\hline N87 & -2.2914322480 & 1.6918403091 & -2.8305734755 \\
\hline N88 & -0.4219645645 & -3.5664660176 & -1.7868233748 \\
\hline N89 & -0.4903632910 & -2.1739408468 & 1.1838290102 \\
\hline N90 & 1.8777272505 & -1.6308505219 & -0.4445395926 \\
\hline N91 & 3.2928120949 & 0.7443951709 & -2.1711652948 \\
\hline C92 & 0.3015870995 & -0.7854559896 & -3.8546849818 \\
\hline C93 & -0.7854378329 & -0.7060555517 & -4.7361004917 \\
\hline H94 & -1.7476588765 & -0.3361185875 & -4.3999906969 \\
\hline C95 & -0.6177129084 & -1.1124503698 & -6.0590523965 \\
\hline H96 & -1.4570912321 & -1.0499508453 & -6.7496950498 \\
\hline N97 & 0.5257187806 & -1.5898132451 & -6.5635420370 \\
\hline C98 & 1.5576154842 & -1.6709007668 & -5.7151119222 \\
\hline H99 & 2.4859188956 & -2.0655566822 & -6.1247869876 \\
\hline $\mathrm{C} 100$ & 1.5030583206 & -1.2909804832 & -4.3757034217 \\
\hline H101 & 2.3822965176 & -1.3918086412 & -3.7473981059 \\
\hline C102 & -3.3784557938 & -1.5868353923 & -1.2980240258 \\
\hline C103 & -4.2957566268 & -0.9612348343 & -2.1534589413 \\
\hline H104 & -4.1013202165 & 0.0286133245 & -2.5490831499 \\
\hline C105 & -5.4689563045 & -1.6326627165 & -2.4969657226 \\
\hline H106 & -6.1871768388 & -1.1519188683 & -3.1594080522 \\
\hline N107 & -5.7947682408 & -2.8548911463 & -2.0649802577 \\
\hline C108 & -4.9102281170 & -3.4512851780 & -1.2559660030 \\
\hline H109 & -5.1734835885 & -4.4493216137 & -0.9103468465 \\
\hline C110 & -3.7107059294 & -2.8745518361 & -0.8461208088 \\
\hline H111 & -3.0468479967 & -3.4215786927 & -0.1839814035 \\
\hline C112 & -3.0392159625 & 2.3534409133 & -3.7828318416 \\
\hline C113 & -4.3169132837 & 2.8537744465 & -3.4809867556 \\
\hline H114 & -4.7300950402 & 2.7377916797 & -2.4839937170 \\
\hline C115 & -5.0274250250 & 3.5105116407 & -4.4823510033 \\
\hline H116 & -6.0183768499 & 3.9079890915 & -4.2693210135 \\
\hline N117 & -4.5734384820 & 3.7001588734 & -5.7269846464 \\
\hline C118 & -3.3527624358 & 3.2234162410 & -6.0015912374 \\
\hline H119 & -2.9925698976 & 3.3887849622 & -7.0155599466 \\
\hline C120 & -2.5493671003 & 2.5514230680 & -5.0846093593 \\
\hline H121 & -1.5659153621 & 2.1863443472 & -5.3619036608 \\
\hline
\end{tabular}




$\begin{array}{llll}\text { C122 } & -0.6439260462 & -4.7724714963 & -2.4196200238 \\ \text { C123 } & -0.7250716728 & -5.9690531404 & -1.6883177850 \\ \text { H124 } & -0.6159094194 & -5.9612369821 & -0.6082717555 \\ \text { C125 } & -0.9501728906 & -7.1556096522 & -2.3813886034 \\ \text { H126 } & -1.0190026118 & -8.0950897981 & -1.8354171055 \\ \text { N127 } & -1.0939371508 & -7.2360410767 & -3.7095728406 \\ \text { C128 } & -1.0137625676 & -6.0883862129 & -4.3951326705 \\ \text { H129 } & -1.1328218994 & -6.1673642693 & -5.4743648541 \\ \text { C130 } & -0.7931249231 & -4.8427181479 & -3.8146516492 \\ \text { H131 } & -0.7370514736 & -3.9431305991 & -4.4191999024 \\ \text { C132 } & -0.6567981332 & -3.4405083560 & 1.8258116035 \\ \text { C133 } & -1.8456186689 & -3.8081784781 & 2.4702076842 \\ \text { H134 } & -2.6867451846 & -3.1264700046 & 2.5158260793 \\ \text { C135 } & -1.9312999658 & -5.0651854868 & 3.0687174768 \\ \text { H136 } & -2.8523595212 & -5.3572714956 & 3.5705969890 \\ \text { N137 } & -0.9449668698 & -5.9674114794 & 3.0777164955 \\ \text { C138 } & 0.1889005110 & -5.6093830292 & 2.4626984148 \\ \text { H139 } & 0.9935517130 & -6.3426071384 & 2.4750891003 \\ \text { C140 } & 0.3832175306 & -4.3844796306 & 1.8291704693 \\ \text { H141 } & 1.3330034006 & -4.1691601920 & 1.3493584265 \\ \text { C142 } & 3.0155165956 & -2.4711414699 & -0.6449911988 \\ \text { C143 } & 2.9027931726 & -3.7892719950 & -1.1078208315 \\ \text { H144 } & 1.9335654254 & -4.2156940227 & -1.3392815471 \\ \text { C145 } & 4.0599344048 & -4.5485795678 & -1.2826281878 \\ \text { H146 } & 3.9784085233 & -5.5726340271 & -1.6432446735 \\ \text { N147 } & 5.2983086906 & -4.1077940678 & -1.0395024853 \\ \text { C148 } & 5.4001063888 & -2.8481028395 & -0.5986472161 \\ \text { H149 } & 6.4093827158 & -2.4874166903 & -0.4068090172 \\ \text { C150 } & 4.3143092234 & -2.0033127711 & -0.3821987165 \\ \text { H151 } & 4.4791632858 & -0.9934140521 & -0.0204741125 \\ \text { C152 } & 4.4690204915 & 0.9683479347 & -2.8567054920 \\ \text { C153 } & 4.5030799149 & 0.9717767014 & -4.2610826112 \\ \text { H154 } & 3.5976859943 & 0.7923042403 & -4.8317023260 \\ \text { C155 } & 5.7203268288 & 1.2042167297 & -4.8958177009 \\ \text { H156 } & 5.7698797448 & 1.2091679813 & -5.9834385808 \\ \text { N157 } & 6.8731950237 & 1.4305146523 & -4.2545166764 \\ \text { C158 } & 6.8278336067 & 1.4298208632 & -2.9164975884 \\ \text { H159 } & 7.7708222058 & 1.6189066470 & -2.4063252191 \\ \text { C160 } & 5.6725202591 & 1.2085440991 & -2.1720324171 \\ \text { H161 } & 5.6936168109 & 1.2296742195 & -1.0872442333\end{array}$

Table S6. Calculated coordinates for neutral decamethylcobaltocene.

$\begin{array}{llll}\text { Co1 } & 0.0004455893 & -1.5503681848 & -0.2915052919 \\ \text { C2 } & 2.5265748421 & -2.4800261974 & -2.2281060131 \\ \text { C3 } & 1.0993428086 & -2.0229141990 & -2.1311961387 \\ \text { C4 } & 0.6506560917 & -0.6576885216 & -2.1093677931 \\ \text { C5 } & -0.0840509228 & -4.3448190778 & -1.9995188407 \\ \text { C6 } & 1.5292912836 & 0.5529445722 & -2.2523203866 \\ \text { C7 } & -0.7934275759 & -0.6453617211 & -2.1298017053 \\ \text { C8 } & -1.6575101107 & 0.5791204287 & -2.2266868944\end{array}$




\begin{tabular}{|c|c|c|c|}
\hline C9 & -1.2270976343 & -1.9871483693 & -2.0249470239 \\
\hline $\mathrm{C} 10$ & -2.6459686340 & -2.4783118617 & -2.0141318910 \\
\hline C11 & -0.0519617745 & -2.8430763222 & -2.0094220631 \\
\hline $\mathrm{C} 12$ & 2.6427667030 & -0.6127356446 & 1.4387989402 \\
\hline $\mathrm{C} 13$ & 1.2253013975 & -1.1094131625 & 1.4451387259 \\
\hline $\mathrm{C} 14$ & 0.7978040881 & -2.4535724270 & 1.5468210869 \\
\hline $\mathrm{C} 15$ & 0.0686241862 & 1.2425724731 & 1.4192808077 \\
\hline $\mathrm{C} 16$ & 1.6688279676 & -3.6735450994 & 1.6402269578 \\
\hline C17 & -0.6462267189 & -2.4486276238 & 1.5244755610 \\
\hline C18 & -1.5185859669 & -3.6639322319 & 1.6651384020 \\
\hline C19 & -1.1014967415 & -1.0855725917 & 1.5467272154 \\
\hline $\mathrm{C} 20$ & -2.5309713794 & -0.6345146086 & 1.6376329433 \\
\hline $\mathrm{C} 21$ & 0.0460183479 & -0.2593562354 & 1.4282832253 \\
\hline $\mathrm{H} 22$ & 0.8472062031 & -4.7670737358 & -1.6083496672 \\
\hline $\mathrm{H} 23$ & -0.2249154640 & -4.7482851102 & -3.0121120204 \\
\hline $\mathrm{H} 24$ & -0.9052449384 & -4.7286432166 & -1.3847378633 \\
\hline $\mathrm{H} 25$ & -2.7703225488 & -3.3383467263 & -1.3473338635 \\
\hline $\mathrm{H} 26$ & -2.9677722570 & -2.7984642137 & -3.0149749450 \\
\hline $\mathrm{H} 27$ & -3.3406573931 & -1.7002872733 & -1.6821810659 \\
\hline $\mathrm{H} 28$ & -1.2101298309 & 1.4338333489 & -1.7073139004 \\
\hline $\mathrm{H} 29$ & -2.6471204270 & 0.4121057611 & -1.7888108935 \\
\hline H30 & -1.8126515867 & 0.8841332199 & -3.2715276328 \\
\hline H31 & 2.5160759309 & 0.3931351814 & -1.8052514049 \\
\hline H32 & 1.0871187025 & 1.4336128012 & -1.7744777728 \\
\hline H33 & 1.6901974259 & 0.8060311587 & -3.3100498688 \\
\hline H34 & 3.2078094510 & -1.7894715260 & -1.7190686536 \\
\hline H35 & 2.8610685796 & -2.5493840778 & -3.2733269635 \\
\hline H36 & 2.6673321776 & -3.4685272990 & -1.7784074102 \\
\hline H37 & -1.0651765674 & -4.5443300333 & 1.1976049505 \\
\hline H38 & -2.5015901317 & -3.5138657610 & 1.2063999924 \\
\hline H39 & -1.6894775693 & -3.9114095763 & 2.7225752440 \\
\hline $\mathrm{H} 40$ & 2.6603154040 & -3.4961781835 & 1.2107071632 \\
\hline H41 & 1.2309212853 & -4.5270683541 & 1.1108279855 \\
\hline $\mathrm{H} 42$ & 1.8189237571 & -3.9862498136 & 2.6835844239 \\
\hline $\mathrm{H} 43$ & 3.3357857974 & -1.3752086644 & 1.0693143388 \\
\hline H44 & 2.9750796575 & -0.3317972979 & 2.4483035695 \\
\hline $\mathrm{H} 45$ & 2.7587908158 & 0.2735735400 & 0.8055715407 \\
\hline H46 & 0.8795951016 & 1.6329388525 & 0.7949661581 \\
\hline H47 & 0.2188305612 & 1.6460851491 & 2.4304403027 \\
\hline $\mathrm{H} 48$ & -0.8702429040 & 1.6583958173 & 1.0397369739 \\
\hline H49 & -2.6791772907 & 0.3380827330 & 1.1569051564 \\
\hline $\mathrm{H} 50$ & -2.8600115320 & -0.5331185586 & 2.6819610940 \\
\hline H51 & -3.2117403149 & -1.3444557847 & 1.1552166779 \\
\hline
\end{tabular}

Table S7. Calculated coordinates for the decamethylcobaltocenium cation.

$\begin{array}{llll}\text { Co1 } & 0.0014421351 & -1.5509343939 & -0.2918159244 \\ \text { C2 } & 2.5353852039 & -2.4477958098 & -2.1062280597 \\ \text { C3 } & 1.1103524089 & -1.9934118220 & -2.0087476733 \\ \text { C4 } & 0.6562120778 & -0.6306170157 & -2.0533595829 \\ \text { C5 } & -0.0339023382 & -4.3436646988 & -1.9354417440\end{array}$




\begin{tabular}{|c|c|c|c|}
\hline C6 & 1.5263758862 & 0.5809253131 & -2.2031713838 \\
\hline $\mathrm{C} 7$ & -0.7795833560 & -0.6399670829 & -2.0053144468 \\
\hline $\mathrm{C} 8$ & -1.6734047654 & 0.5599689633 & -2.0979172015 \\
\hline C9 & -1.2124914011 & -2.0082310270 & -1.9295748830 \\
\hline $\mathrm{C} 10$ & -2.6344695673 & -2.4812231207 & -1.9286259144 \\
\hline $\mathrm{C} 11$ & -0.0444061870 & -2.8446994730 & -1.9326386249 \\
\hline $\mathrm{C} 12$ & 2.6464311422 & -0.6578650826 & 1.3564561003 \\
\hline C13 & 1.2175729851 & -1.1104118472 & 1.3506478180 \\
\hline $\mathrm{C} 14$ & 0.7648646589 & -2.4721074491 & 1.4242345574 \\
\hline $\mathrm{C} 15$ & 0.0722083474 & 1.2410823279 & 1.3492764469 \\
\hline $\mathrm{C} 16$ & 1.6380598192 & -3.6869092395 & 1.5189145163 \\
\hline $\mathrm{C} 17$ & -0.6709045101 & -2.4607287528 & 1.4689004151 \\
\hline $\mathrm{C} 18$ & -1.5569359928 & -3.6606765247 & 1.6181249610 \\
\hline C19 & -1.1054496129 & -1.0923598433 & 1.4214886301 \\
\hline $\mathrm{C} 20$ & -2.5235914187 & -0.6165637457 & 1.5120365175 \\
\hline $\mathrm{C} 21$ & 0.0615743853 & -0.2577218791 & 1.3483770978 \\
\hline $\mathrm{H} 22$ & 0.8622889543 & -4.7479192423 & -1.4568810410 \\
\hline $\mathrm{H} 23$ & -0.0471976850 & -4.7169939041 & -2.9675255398 \\
\hline $\mathrm{H} 24$ & -0.9091497872 & -4.7599708688 & -1.4294437697 \\
\hline $\mathrm{H} 25$ & -2.7480492407 & -3.4317834753 & -1.4008666850 \\
\hline $\mathrm{H} 26$ & -2.9787753117 & -2.6382030513 & -2.9589314965 \\
\hline $\mathrm{H} 27$ & -3.3081890058 & -1.7524046340 & -1.4698056252 \\
\hline $\mathrm{H} 28$ & -1.1908247982 & 1.4588179895 & -1.7052168300 \\
\hline $\mathrm{H} 29$ & -2.6139005440 & 0.4136478406 & -1.5597101077 \\
\hline $\mathrm{H} 30$ & -1.9271882369 & 0.7601533062 & -3.1472611901 \\
\hline H31 & 2.5071478358 & 0.4398472934 & -1.7406578368 \\
\hline H32 & 1.0671053690 & 1.4711272477 & -1.7650906262 \\
\hline H33 & 1.6965706329 & 0.7927270948 & -3.2669024478 \\
\hline H34 & 3.2259420074 & -1.7200544269 & -1.6717752188 \\
\hline H35 & 2.8183154109 & -2.5757588296 & -3.1593356530 \\
\hline H36 & 2.6935118656 & -3.4088621227 & -1.6094385414 \\
\hline H37 & -1.1147482411 & -4.5544431534 & 1.1695528079 \\
\hline H38 & -2.5400231268 & -3.5022332003 & 1.1666049623 \\
\hline H39 & -1.7196414928 & -3.8780279535 & 2.6818523341 \\
\hline $\mathrm{H} 40$ & 2.5963261391 & -3.5427429268 & 1.0125433968 \\
\hline H41 & 1.1563026396 & -4.5712175071 & 1.0929135237 \\
\hline H42 & 1.8570889028 & -3.9119077770 & 2.5708695073 \\
\hline $\mathrm{H} 43$ & 3.3082407010 & -1.3909232059 & 0.8876969869 \\
\hline H44 & 2.9934942470 & -0.5198082610 & 2.3887739186 \\
\hline $\mathrm{H} 45$ & 2.7754345498 & 0.2979349892 & 0.8413051569 \\
\hline H46 & 0.9527917464 & 1.6440995989 & 0.8419190150 \\
\hline H47 & 0.0912866292 & 1.6156581956 & 2.3807499702 \\
\hline H48 & -0.8184561934 & 1.6571519369 & 0.8704863540 \\
\hline H49 & -2.6670609047 & 0.3423829933 & 1.0069252152 \\
\hline H50 & -2.8060229704 & -0.4754140137 & 2.5631675187 \\
\hline H51 & -3.2242975795 & -1.3377359117 & 1.0829272824 \\
\hline
\end{tabular}

